\title{
Eye-specific segregation and differential fasciculation of developing retinal ganglion cell axons in the mouse visual pathway
}

\author{
Austen A. Sitko ${ }^{1,}$, Takaaki Kuwajima ${ }^{1,2}$, and Carol Mason ${ }^{1,2}$ \\ ${ }^{1}$ Departments of Neuroscience, and Ophthalmology, College of Physicians and Surgeons, \\ Columbia University, New York, New York, 10032 \\ ${ }^{2}$ Pathology and Cell Biology, and Ophthalmology, College of Physicians and Surgeons, Columbia \\ University, New York, New York, 10032
}

\section{Abstract}

Prior to forming and refining synaptic connections, axons of projection neurons navigate long distances to their targets. While much is known about guidance cues for axon navigation through intermediate choice points, whether and how axons are organized within tracts is less clear. Here we analyze the organization of retinal ganglion cell (RGC) axons in the developing mouse retinogeniculate pathway. RGC axons are organized by both eye-specificity and topography in the optic nerve and tract: ipsilateral RGC axons are segregated from contralateral axons and are offset laterally in the tract relative to contralateral axon topographic position.

To identify potential cell-autonomous factors contributing to the segregation of ipsilateral and contralateral RGC axons in the visual pathway, we assessed their fasciculation behavior in a retinal explant assay. Ipsilateral RGC neurites self-fasciculate more than contralateral neurites in vitro and maintain this difference in the presence of extrinsic chiasm cues.

To further probe the role of axon self-association in circuit formation in vivo, we examined RGC axon organization and fasciculation in an EphB1 ${ }^{-/-}$mutant, in which a subset of ipsilateral RGC axons aberrantly crosses the midline but targets the ipsilateral zone in the dorsal lateral geniculate nucleus on the opposite side. Aberrantly crossing axons retain their association with ipsilateral axons in the contralateral tract, indicating that cohort-specific axon affinity is maintained independently of guidance signals present at the midline. Our results provide a comprehensive assessment of RGC axon organization in the retinogeniculate pathway and suggest that axon selfassociation contributes to pre-target axon organization.

\section{Keywords}

Retinal ganglion cell; axon guidance; fasciculation; visual system; development; axon; RRID:IMSR_JAX:000664; RRID:SCR_001775; RRID:SCR_002285; RRID:SCR_008058;

\footnotetext{
*Current address, corresponding author: Austen A. Sitko, Department of Neurobiology, Harvard Medical School, 456 Goldenson, 220 Longwood Avenue, Boston, MA 02115, austen_sitko@hms.harvard.edu.

Conflict of Interest: The authors declare no competing financial interests.
} 
RRID:SCR_008624; RRID:SCR_002798; RRID: AB_2491179; RRID: AB_531793; RRID:

AB_143165; RRID: AB_2534073; RRID: AB_2534088

\section{Introduction}

A guiding principle in neural development and axon guidance is the relationship between the organization of neuronal cell bodies and their axonal projections in targets. In the visual system, for instance, neighboring retinal ganglion cells (RGCs) in the retina project onto neighboring relay cells in thalamic targets, which in turn project onto similarly positioned target cells in the visual cortex. With this emphasis on source-target correspondence, less attention has been given to the organization of axons within developing tracts, which may be important for establishing appropriate order in connections within targets. Open questions include whether defined order exists among axons prior to target entry, what interactions occur between and within axon cohorts to generate such order, and what bearing pre-target axon organization has on targeting.

Three organizational modes have been identified in axon tracts in several systems and species: topography, typography, and chronotopy (reviewed in Sitko \& Mason, 2015). Topography, based on the spatial arrangement of neurons, is the most commonly identified organizational feature in neural circuits and axon tracts. In the visual system, topographic axon order in the optic nerve and tract reflects the retinotopic position of RGCs, which is then mapped onto the dorsal lateral geniculate (dLGN), their thalamic target (e.g., Chan \& Chung, 1999; Plas, Lopez, \& Crair, 2005; Torrealba, Guillery, Eysel, Polley, \& Mason, 1982). Typography is based on neuron subtype or cohort-specific molecular expression. In the olfactory nerve, for instance, molecules such as neuropilin-1 (Nrp-1) and neural cell adhesion molecule (NCAM) contribute to olfactory sensory axon organization prior to glomerular targeting, which is based on olfactory receptor expression (Miller, Maurer, Zou, Firestein, \& Greer, 2010). Finally, chronotopy is based on axon age and time of entry into the tract. In the visual system, younger RGC axons grow along older axons in a sequentially additive fashion in the optic tract (Walsh \& Guillery, 1985). In all major sensory axon tracts studied to date, one or more of these three modes of axon organization have been identified (Sitko \& Mason, 2015).

Target-removal experiments in the olfactory and visual systems of mice and frogs demonstrated that axons develop distinct organization in their tracts independently of targetderived cues (Graziadei, Levine, \& Graziadei, 1978; Reh, Pitts, \& Constantine-Paton, 1983; St John, Clarris, McKeown, Royal, \& Key, 2003). Furthermore, disturbing pre-target axon organization in the tract can affect targeting. For instance, perturbations in Nrp-1 and Semaphorin expression disrupt pre-target axon sorting in the olfactory nerve (Imai et al., 2009) and corpus callosum (Zhou et al., 2013), which lead to subsequent shifts in axon targeting in the olfactory bulb and contralateral cortex, respectively.

Our understanding of the arrangement of RGC axons in the retinogeniculate pathway remains incomplete, especially with regard to how two modes of pre-target organization, topography and typography, relate to one another. RGC axons project either ipsi- or contralaterally at the optic chiasm, and their terminals are organized based on retinotopy and 
laterality (i.e., eye-specific ipsilateral- and contralateral-recipient zones) in the dLGN. Studies have described varying degrees of topographic order of RGC axons in the rodent optic nerve and tract (Chan \& Chung, 1999; Chan \& Guillery, 1994; Plas et al., 2005), but only one study addressed eye-specific organization of ipsilateral and contralateral RGC axons in the optic tract (Godement, Salaun, \& Imbert, 1984).

We therefore aimed to provide a comprehensive assessment of RGC axon order along the pathway to the dLGN and to identify possible mechanisms of axon organization. By examining both topographic and eye-specific RGC axon organization in the mouse retinogeniculate pathway, we provide the first direct comparison, to our knowledge, of two distinct modes of axon organization in a sensory axon tract. Additionally, we provide evidence, both in vitro and in vivo, for differential self-fasciculation and selective axon coherence as mechanisms of tract organization that could contribute to the establishment of accurate neural circuits. Our results are consistent with a model in which maintenance of organizational continuity through the pathways of projection axons is an important feature of developing circuits.

\section{Materials and Methods}

\section{Animals}

Mice were maintained in a timed-pregnancy colony in a barrier facility at Columbia University Medical Center. All procedures were carried out in accordance with Columbia University Institutional Animal Care and Use Committee. Breeding females were checked for vaginal plug at noon each weekday; the day on which a vaginal plug was detected was considered embryonic day (E) 0.5. Embryos were harvested as close to noon on the day of collection as possible. Wild-type animals were C57BL/6J (RRID:IMSR_JAX:000664). ET33 SERT-Cre mice were generated by GENSAT (Gong et al., 2007); ET33 SERTCre::zsGreen mice were provided as a gift from the T. Maniatis Lab (Columbia University) and maintained on a C57BL/6J background. EphB1 ${ }^{-/-}$mice, described previously (Williams et al., 2003), were maintained on a 129S1/SvImJ background. SERT-Cre::zsGreen mice were crossed with EphB1 $1^{-/}$mutants and were bred for three generations before collecting litters of SERT-Cre::zsGreen;EphB1 (SERT;EphB1) offspring. All experiments using SERT;EphB1 animals compared littermates with SERT-Cre::zsGreen ${ }^{+/-}$and EphB1 ${ }^{-/-}$, EphB1 $1^{+/+}$, or EphB1 $1^{+/-}$.

\section{Anterograde axon labeling}

Anterograde labeling was performed with DiI (1,1' -Dioctadecyl-3,3,3', $3^{\prime}$ -

Tetramethylindocarbocyanine Perchlorate (DiIC18(3))) and its far red shifted analogue, DiD (1,1'-Dioctadecyl-3,3,3', $3^{\prime}$-Tetra- methylindodicarbocyanine, 4-Chlorobenzenesulfonate Salt) (Molecular Probes, Cat\# D282 and D7757, respectively). Embryos were harvested from pregnant dams anesthetized with ketamine/xylazine (100 and $10 \mathrm{mg} / \mathrm{kg}$, respectively, in $0.9 \%$ saline), transcardially perfused with $4 \%$ paraformaldehyde (PFA) in $1 \mathrm{X}$ phosphate buffered saline (PBS), and post-fixed overnight $(\mathrm{O} / \mathrm{N})$ at $4^{\circ} \mathrm{C}$. Postnatal pups were anesthetized with ketamine/xylazine (as for pregnant dams), transcardially perfused with $4 \%$ 
PFA, and post-fixed $\mathrm{O} / \mathrm{N}$ at $4^{\circ} \mathrm{C}$. Perfused heads were rinsed twice in $1 \mathrm{X}$ PBS before labeling.

For whole eye anterograde labeling, the eyecup was removed and the optic nerve head was gently blotted with a Kimwipe to remove excess moisture. DiI or DiD crystals were placed directly onto the optic nerve head using the fine tip of a pulled glass micropipette, and crushed into the optic nerve head to ensure full labeling of retinal axons. The eyecup was replaced to hold DiI or DiD in place during incubation. For focal anterograde labeling (ventrotemporal or topographic label), the lens was removed, leaving the eyecup intact, and the retina was gently blotted with a Kimwipe to remove excess moisture. The tip of a pulled glass micropipette was used to place a small amount of DiI or DiD into the designated peripheral retinal quadrant, ensuring penetration of the RGC layer. The lens was gently replaced to keep the DiI or DiD in place during incubation. Embryonic heads were incubated at $37^{\circ} \mathrm{C}$ for 6-12 days; postnatal heads were incubated at $37^{\circ} \mathrm{C}$ for $10-14$ days.

After incubation to allow for dye transport, brains were removed and sectioned with a vibratome at $75 \mu \mathrm{m}$ in either the frontal or horizontal plane, and mounted sequentially in Fluoro-Gel mounting medium (Electron Microscopy Sciences). Retinae with focal labeling were removed after cutting a slit into the ventral retina for orientation, then flat-mounted and coverslipped with Fluor-Gel mounting medium in order to image for assessment of dye placement and extent of labeling.

\section{Retinal explant cultures}

Retinal explant procedures have been described previously (Kuwajima et al., 2012; Petros, Bryson, \& Mason, 2010). Before plating explants, glass-bottomed culture dishes were incubated $\mathrm{O} / \mathrm{N}$ at $4^{\circ} \mathrm{C}$ with $0.01 \%$ poly-DL-ornithine solution (Sigma Cat\# P4957), rinsed the next day three times with cold DMEM/F12, and incubated at $37^{\circ} \mathrm{C}$ for at least 2 hours (h) with $20 \mu \mathrm{g} / \mathrm{ml}$ laminin (Invitrogen Cat\# 23017-015). On the experimental day, a pregnant dam at E14.5 was anesthetized with ketamine/xylazine (100 and $10 \mathrm{mg} / \mathrm{kg}$, respectively, in $0.9 \%$ saline) and sacrificed by cervical dislocation. Embryos were collected into cold DMEM/F12 and decapitated using forceps. Heads were placed into a fresh dish of cold DMEM/F12 and microscissors were used to dissect each eye of each head after making a small cut into the ventral (V) retina to indicate orientation. The lens and interior vasculature were removed and eyecups collected into cold DMEM/F12. Each retina was then dissected into explants approximately $300 \times 300 \mu \mathrm{m}$, collecting ventrotemporal (VT) and dorsotemporal (DT) explants separately. Explants were plated in serum free media (SFM) $+0.4 \%$ methylcellulose (Sigma catalog no. M-0512). SFM is composed of 1\% (weight/ volume) fatty acid free bovine serum albumin (BSA, Sigma Cat\# A8806-5G; n.b. other BSA affect neurite outgrowth and fasciculation), $1 \%$ (volume/volume) ITS supplement (Sigma Cat\# I-1884), and 0.2\% (volume/volume) Pen/Strep (Invitrogen Cat\# 15140-122) in DMEM/F12 media; after mixing ingredients at $4^{\circ} \mathrm{C} \mathrm{O} / \mathrm{N}$, solution was filtered with a $0.2 \mu \mathrm{m}$ syringe filter.

Three explants of the same type (i.e., VT or DT) were plated per dish in SFM + 0.4\% methylcellulose, spaced equally far apart from each other. Explants with neurites that touched in the culture dish were excluded from analysis. For chiasm co-cultures, the chiasm 
region was dissected from the same embryonic heads that provided retinae, and cells were dissociated as in Kuwajima et al. (2012). Dissociated chiasm cells were plated at a density of $3.5 \times 10^{5} \mathrm{cells} / \mathrm{ml}$ and allowed to adhere to the laminin-coated dish for at least $1 \mathrm{~h}$ at $37^{\circ} \mathrm{C}$ before plating explants. Plated explants were incubated $\mathrm{O} / \mathrm{N}$ at $37^{\circ} \mathrm{C}$ and fixed the following day for 15-20 minutes (min) with warm 4\% PFA, then rinsed with $1 \mathrm{X}$ PBS and immunostained.

Analysis of neurite bundle widths was performed with Neurolucida software. Explants with marked asymmetry in neurite outgrowth were excluded from analysis, as were those with an explant body radius outside of a 100-200 $\mu \mathrm{m}$ range. For neurite fasciculation analysis, explant body radius was measured and two rings were placed around the neurite halo, positioned at radial distances of $250 \mu \mathrm{m}$ and $500 \mu \mathrm{m}$ from the edge of the explant. High magnification (40X) tiled images were collected around each ring for analysis. The width of each neurite or neurite bundle intersecting each ring was manually traced in Neurolucida, and data exported into .csv files from Neurolucida Explorer, and then analyzed with python (see Experimental Design and Statistical Analysis for details).

\section{Immunohistochemistry}

SERT-Cre::zsGreen heads were cryosectioned $25 \mu \mathrm{m}$ thick, collected in sequence in four parallel series. One or two sets of slides were immunostained, for serial sections either 100 $\mu \mathrm{m}$ or $50 \mu \mathrm{m}$ apart, respectively. Slides were incubated for 45-90 $\mathrm{min}$ at room temperature (RT) with blocking solution composed of $1 \mathrm{X}$ PBS $+0.5 \%$ Tween $+10 \%$ normal goat serum (NGS). Primary antibodies (see Table 1) against zsGreen and neurofilament (NF) were applied in a $1 \mathrm{X}$ PBS $+0.5 \%$ Tween $20+10 \%$ NGS solution and incubated for 2 nights at $4^{\circ}$ C. Samples were then rinsed for 15-20 min three times with 1X PBS, and secondary antibodies (goat anti-rabbit Alexa Fluor 488 and goat anti-mouse IgG Alexa Fluor 594, see Table 1 for details) were applied at 1:400 in 1X PBS + 0.5\% Tween $20+5 \%$ NGS for 3-4 h at RT. Samples were rinsed once with 1X PBS, incubated with Hoechst (Thermo Fisher Cat\# $62249,1: 2,000$ in 1X PBS) at RT for 10-20 min, rinsed three times with 1X PBS, and coverslipped with Fluoro-Gel mounting medium.

Explant cultures were fixed with warm 4\% PFA in 1X PBS for 15-20 min at RT, and rinsed 2-3 times with RT 1X PBS prior to immunostaining. Fixed cultures were incubated for 45-90 min at RT in blocking solution composed of $1 \mathrm{X}$ PBS $+1 \%$ Triton X-100 + 10\% NGS. Primary antibody against NF (see Table 1 for details) was applied in a $1 \mathrm{X}$ PBS $+1 \%$ Triton $\mathrm{X}-100+5 \%$ NGS solution and incubated $\mathrm{O} / \mathrm{N}$ at $4{ }^{\circ} \mathrm{C}$. The following day, samples were rinsed for 15-30 min three times with $1 \mathrm{X}$ PBS at RT. Secondary antibody (goat anti-mouse Alexa Fluor 488, see Table 1 for details) was applied at 1:400 in a 1X PBS + 1\% Triton $\mathrm{X}-100+5 \%$ NGS solution and incubated at RT for 2-4 h. For chiasm co-cultures, Hoechst nuclear stain was applied as above. Samples were rinsed three times and coverslips mounted onto slides with Fluoro-Gel mounting medium.

\section{Imaging}

Imaging was performed using a Zeiss AxioImager M2 microscope with Apotome, AxioCam MRm camera, and Neurolucida software (v11, MBF Biosciences, Williston, VT, USA, 
RRID:SCR_001775); with a 5X objective lens (Fluar, NA $=0.25$, working distance $=12.5$ $\mathrm{mm}$ ), 20X objective lens (Plan-APO, NA $=0.8$, working distance $=550 \mu \mathrm{m}$ ), or 40X objective lens (Plan-APO, NA $=1.4$, working distance $=130 \mu \mathrm{m}$ ). Images were acquired in MBF.tiff format and processed in Fiji (ImageJ, NIH, RRID:SCR_002285). Images in Fig. 2e-f and Movies 1 and 2 were acquired in the Confocal and Specialized Microscopy Shared Resource of the Herbert Irving Comprehensive Cancer Center at Columbia University, using Nikon Ti Eclipse inverted A1 confocal microscope with Plan-Fluor 40X Oil objective $(\mathrm{NA}=1.3)$ and Nikon NIS Elements software. Images were processed in Fiji, brightness and contrast were adjusted for presentation in figures.

\section{Experimental Design and Statistical Analysis}

Analysis of zsGreen+ ipsilateral RGC axons in the SERT-Cre::zsGreen reporter mouse (Fig. 1) was performed in four or more animals from three or more litters, for both frontal and horizontal sections. Assessment of eye-specific RGC axon organization via DiI/DiD anterograde labeling (Fig. 2-3) was performed in at least five animals from at least three litters, per age and condition (i.e., whole eye or VT-specific labeling). Similarly, topographic labeling via DiI/DiD anterograde labeling was assessed in at least four animals from three litters for each hemiretina labeling, and for each smaller quadrant labeling, in at least three animals from three litters. Anterograde labeling with $\mathrm{DiI}$ and $\mathrm{DiD}$, particularly for focal labeling of the retina, is inherently variable. Therefore, brains with incomplete or faint labeling from either DiI or DiD were excluded from analysis. In all cases, male and female littermates were analyzed together.

In vitro experiments of fasciculation (Fig. 6, Fig. 7k-o) were performed in triplicate, with near equal numbers of explants in each condition for each experimental replication (DT, VT, $\mathrm{DT}+\mathrm{OC}, \mathrm{VT}+\mathrm{OC}$, and, separately, EphB1 ${ }^{-/-} \mathrm{DT}$ and $\left.\mathrm{EphB} 1^{-/-} \mathrm{VT}\right)$. For each experiment, retinae were pooled from one litter, using all embryos and combining males and females. For co-cultures of retinal explants and dissociated optic chiasm cells, chiasm cells were pooled from the entire litter. Specific values for numbers of explants analyzed are listed in Results and figure legends, with the $\mathrm{p}$ values for each experiment and analysis. Graphing and statistical analysis of cumulative frequency distribution plots for the in vitro assay of fasciculation (Fig. 6c-d, g, Fig. 7n-o) were performed in python. The scipy.stats package was used to run the Mann-Whitney $U$ test to compare cumulative distribution frequencies between explant types and conditions (SciPy, http://www.scipy.org, RRID:SCR_008058). The Matplotlib.pyplot package was used to plot cumulative distribution frequencies (MatPlotLib, http://matplotlib.sourceforge.net, RRID:SCR_008624). Graphing and statistical analysis for explant size and neurite outgrowth (Fig. 6e-f, Fig. 7l-m) were performed in GraphPad Prism7 (v7, GraphPad Software, La Jolla, California, www.graphpad.com, RRID:SCR_002798), using one-way ANOVA with the Holm-Sidak's multiple comparisons test when appropriate. P values are reported in the text in the Results section and figure legends.

Assessment of anterogradely labeled RGC axons in the EphB1 $1^{-/-}$mutant (Fig. 7c-d) was conducted on at least five animals from three litters, again pooling male and female pups. Analysis of zsGreen+ cell numbers in the retina (Fig. 7f-g) and axon distribution in the tract 
(Fig. 7h-j) in SERT;EphB1 mice was performed on littermates with EphB1 $1^{+/+}$, EphB1 ${ }^{+/-}$, and $\mathrm{EphB} 1^{-/-}$genotypes. Imaging and analyses were performed blinded to genotype. For quantification of the percent of tract width occupied by zsGreen+ axons, measurements were taken from three sequential sections, $100 \mu \mathrm{m}$ apart, beginning at the first section caudal to the chiasm in which the chiasm was no longer visible. For each animal, one hemisphere was arbitrarily chosen to analyze and measurements were taken at the widest part of the tract in each section. Data analysis of zsGreen+ cell numbers in the retina and zsGreen+ axon distribution in the tract of SERT;EphB1 mice were performed in GraphPad Prism7 using one-way ANOVA with the Holm-Sidak's multiple comparisons test. P values are reported in the text in the Results section and figure legends.

\section{Results}

\section{Ipsilateral RGC axons are positioned laterally in the optic nerve and tract}

Godement et al. (1984) used horseradish peroxidase labeling of one eye to show that ipsilateral RGC axons were situated laterally in the postnatal murine optic tract. Such anterograde labeling, however, precluded tracing ipsilateral and contralateral axons in the optic nerve. Therefore, we used genetic labeling of ipsilateral RGCs in the SERTCre::zsGreen reporter mouse to track ipsilateral axon position along the full extent of the retinogeniculate pathway (see Methods). Zic2, the ipsilateral-RGC-specific transcription factor, directly regulates expression of the serotonin transporter (SERT) (Garcia-Frigola \& Herrera, 2010). SERT is important for eye-specific segregation in the dLGN and superior colliculus (SC), but is not involved in ipsilateral RGC axon choice at the midline optic chiasm (Garcia-Frigola \& Herrera, 2010; Salichon et al., 2001; Upton et al., 1999). SertCre::zsGreen expression allowed us to track ipsilateral axons before they diverge at the optic chiasm, at which point they become anatomically distinguishable by classic anterograde labeling methods.

SERT-Cre::zsGreen mice have robust zsGreen expression restricted to the ventrotemporal (VT) retina (Fig. 1a), the source of ipsilateral RGCs (Colello \& Guillery, 1990; Godement, Salaun, \& Mason, 1990). This expression pattern is consistent with SERT-Cre expression patterns shown previously (Koch et al., 2011) and SERTmRNA expression shown by Garcia-Frigola and Herrera (2010), which corresponds in turn to Zic2 mRNA expression (Herrera et al., 2003). Figure 1 shows SERT-Cre::zsGreen labeling of ipsilateral RGC axons in frontal (Fig. 1b-f) and horizontal (Fig. 1g-h) planes from postnatal day (P) 0 mice. The expression of zsGreen, seen in a frontal section of the retina (Fig. 1b), is restricted to the RGC layer, and zsGreen+ RGC axons exit the retina into the ventral portion of the optic nerve (Fig. 1b, lower panels).

Once in the optic nerve, zsGreen+ axons cluster predominantly in a ventrolateral position (Fig. 1c, arrowheads), although there is a secondary cluster of zsGreen+ axons in the medial optic nerve (Fig. 1c, arrows). The second group of zsGreen+ axons appears more loosely bundled than the principal set of zsGreen+ axons in the ventrolateral nerve. This distribution is similar when viewed in the horizontal plane (Fig. 1g): zsGreen+ axons are primarily positioned in the lateral portion of the optic nerve, with some zsGreen+ axons in the medial nerve. The lateral and medial bundles are more obvious in the section dorsal to the optic 
chiasm (Fig. 1g, bottom), highlighting the dorsoventral distribution of zsGreen+ axons that is more readily apparent in the frontal view (Fig. 1c).

As RGC axons near the optic chiasm, zsGreen+ axons appear progressively less tightly bundled together (Fig. 1c, bottom panels, and Fig. 1g, top). Within the optic chiasm, zsGreen + axons span nearly the full dorsoventral extent of the chiasm (vertical lines in Fig. 1d), and are biased rostrally along the rostrocaudal axis (Fig. 1g, top). The images in Fig. 1 are from P0 pups, an age at which the majority of ipsilateral RGC axons have turned away from and extended beyond the optic chiasm. However, axons from the latest-born ipsilateral RGCs are still navigating the chiasm at $\mathrm{P} 0$, and these are likely the sparse zsGreen+ axons in the middle of the chiasm region of the micrographs in Fig. 1d and g.

ZsGreen+ RGC axons in the optic tract are loosely bundled just caudal to the chiasm (Fig. 1e, top panel; Fig. 1g, top panel) and as they progress into the tract, become increasingly well bundled along the lateral side of the tract (Fig. 1e, lower panels, and Fig. 1h). Farther distally, near the dLGN, zsGreen+ RGC axons remain clustered along the lateral edge of the optic tract (Fig. 1f, h). ZsGreen+ axon position in the lateral optic tract does not markedly change along the length of the tract, even as axons approach the dLGN (Fig. 1f, h).

\section{Ipsilateral and contralateral RGC axons are segregated in the optic tract}

Analysis of the SERT-Cre::zsGreen visual system provides a clear view of the position of ipsilateral RGC axons in the optic nerve, chiasm, and tract, and illustrates the previously unappreciated dynamic organization of this cohort of axons. Specifically, ipsilateral axons are well bundled in the proximal optic nerve, less so in the distal nerve, and then proceed from less to more well bundled in the optic tract. However, this genetic labeling does not afford a direct comparison of ipsilateral and contralateral RGC axons.

In order to directly compare the position of these two cohorts of RGC axons in the optic tract, we used classic anterograde tracing with the carbocyanine lipophilic dye tracer DiI and its far-red-shifted analog DiD. We performed two-color DiI and DiD labeling in embryonic and early postnatal wild-type mice (schematized in Fig. 2a) and viewed $75 \mu \mathrm{m}$ sections through the entire optic tract in both frontal (data not shown) and horizontal planes. Fig. 2b shows representative sections in the horizontal plane of the optic tract at select ages from E15 to P2. RGCs extend their axons out of the retina in a central to peripheral wave starting around E12-13 and ending by P0 (Drager, 1985). Thus, the size of the optic tract increases as more axons enter it during late embryogenesis. At E15 and E16, ipsilateral axons from the VT retina have just begun to enter the tract and cluster in the lateral edge of the tract. Other ipsilateral RGC axons are more sparsely positioned in the medial optic tract, but disappear by later embryonic ages. These axons are likely from the earliest-born ipsilateral RGCs in the dorsocentral retina, a transient population (Colello \& Guillery, 1990) whose axons have been found in the medial optic tract (Soares \& Mason, 2015). By E18, as the number of ipsilateral RGC axons in the tract increases, eye-specific segregation of ipsi- and contralateral RGC axons is more evident. The high and low density of ipsilateral and contralateral axons, respectively, in the lateral tract, is most striking at $\mathrm{P} 0$, and continues through P2, when most, if not all, RGC axons have reached the optic tract (Fig. 2b). Moreover, this organization is maintained throughout the full length of the optic tract, even 
as the tract as a whole changes shape, widening and flattening slightly as it nears the LGN (Fig. 2c).

In order to better appreciate the nature of ipsilateral and contralateral axon segregation, we performed high-resolution imaging of the intact $\mathrm{P} 0$ optic tract in a wholemount, labeled as before with DiI and DiD (Fig. 2d), and cleared with Clear $^{T}$ (Kuwajima et al., 2013). In the single optical slices shown in Fig. 2e and $\mathrm{f}$ of the proximal and distal optic tract, respectively, ipsilateral and contralateral axons bundle together to varying extents. Some large bundles of each type are visible (larger puncta in the orthogonal views in Fig. 2e' and f'), and single axons of different types also mingle in close proximity to each other. While individual ipsilateral and contralateral fibers occasionally commingle, on the population level (best appreciated in the orthogonal views), the two cohorts of axons are grossly segregated (see also Movies 1 and 2). The change in shape of the optic tract from the proximal to distal aspect can be seen in the orthogonal views, with the distal tract flattening out (Fig. 2f') as it nears the dLGN, compared with the more rounded proximal tract (Fig. $\left.2 e^{\prime}\right)$.

\section{RGC axons are organized both by laterality and topography in the optic tract}

Because ipsilateral RGCs are topographically restricted to the VT retina (Fig. 3a, left), we next asked if the segregation of ipsilateral and contralateral axons in the optic tract was simply a reflection of topographic organization. In order to distinguish between topographic and typographic (i.e., ipsilateral and contralateral) organizational modes in the optic tract, we took advantage of the fact that VT retina produces both ipsilateral and contralateral RGCs during late embryogenesis (Pak, Hindges, Lim, Pfaff, \& O'Leary, 2004; Williams et al., 2006) (Fig. 3a, right). Focal labeling of the VT region of each retina with DiI or DiD (Fig. 3b) affords a direct comparison of eye-specific axon organization within the same topographic group of RGCs. If RGC axons are organized only by topographic principles, then ipsilateral and contralateral VT axons would be intermingled in the optic tract. If, on the other hand, there is eye-specific axon organization in addition to topographic order, then ipsilateral and contralateral RGC axons emanating from VT retina would segregate in the optic tract.

We analyzed the optic tracts of $\mathrm{P} 0$ and $\mathrm{P} 2$ pups, when late-born VT contralateral axons have had sufficient time to reach the tract. Indeed, ipsilateral and contralateral RGC axons arising from the VT retina are grossly segregated in the optic tract at these ages (Fig. 3c), confirming true eye-specific axon organization. Direct comparison the ipsilateral and contralateral hemispheres following DiI/DiD labeling in the VT retina underscores that, while ipsilateral and contralateral RGC axons from VT retina are positioned in largely the same area of the tract, abiding by topographic order, the two populations are segregated from one another, with ipsilateral axons biased laterally from contralateral axons (Fig. 3d).

These data then raised the question of whether the eye-specific and topographic maps of RGC axons in the retinogeniculate pathway are in agreement. While topographic organization of RGC axons in the optic nerve and tract has been documented previously, there is disagreement over the extent and details of organization among retinofugal axons, in part due to variable and non-standard planes of section and diverse labeling schemes across 
studies. Few reports studied the mouse (Chan \& Chung, 1999; Plas et al., 2005), with many more favoring the visual systems of the rat, ferret, cat, or amphibians (reviewed in Sitko \& Mason, 2015). Furthermore, none of the studies directly compared topographic and eyespecific order in the nerve and tract. Thus, to directly address the extent to which the topographic and eye-specific maps in the optic tract are in register, we performed a series of DiI/DiD two-color anterograde labeling experiments with different combinations of topographic coordinates in the retina at $\mathrm{P} 0$, and assessed axon organization in both horizontal and frontal sections (Fig. 4a).

Hemiretina labeling of opposing topographic poles (dorsal/ventral in Fig. 4b and temporal/ nasal in Fig. 4c) demonstrates consistent topographic organization throughout the retinogeniculate pathway. Ventral RGC axons traverse the ventrolateral aspect of the optic nerve (Fig. $4 \mathrm{~b}^{4}$, top), although there is overlap between dorsal and ventral axons in the nerve (Fig. $4 \mathrm{~b}^{2}$ and $\mathrm{b}^{4}$, top). In the optic chiasm, dorsal axons route largely through the middle of the rostrocaudal aspect of the chiasm, while ventral axons are more evenly distributed across the same axis (Fig. $4 b^{2}$ ). In the optic tract, dorsal axons are positioned in the caudomedial tract, while ventral axons take a rostrolateral position (Fig. $4 b^{2-3}$ and $b^{4}$, bottom). These positions are maintained through the length of the optic tract, although as axons ascend dorsally, the rostrocaudal segregation predominates (Fig. $4 \mathrm{~b}^{3}$ ). Temporal and nasal RGC axons are also consistently organized (Fig. 4c). In the optic nerve, nasal RGC axons are situated dorsomedially and temporal axons are positioned ventrolaterally (Fig. $4 \mathrm{c}^{2}$ and $\mathrm{c}^{4}$, top). This orientation and segregation is preserved through the chiasm (Fig. $4 \mathrm{c}^{2}$ ). In the optic tract, nasal and temporal axons are segregated across the rostrocaudal axis (Fig. $4 c^{2-3}$ and $b^{4}$, bottom).

We next performed smaller focal DiI/DiD anterograde labeling of opposite (dorsal/ventral, nasal/temporal) and adjacent (ventral/temporal) retinal quadrants, in which we directly compared ipsilateral and contralateral RGC axon projections from each topographic region (Fig. 5a-c). Similar to the hemiretina labeling (Fig. 4), focal anterograde tracing demonstrates a rostrolateral to caudomedial segregation of ventral and dorsal RGC axons in the contralateral optic tract (Fig. 5a). The rostrocaudal segregation of nasal and temporal RGC axons in the contralateral optic tract is also evident (Fig. 5b). Additionally, focal anterograde labeling of the adjacent ventral and temporal quadrants of the retina shows a rostrolateral position of ventral axons and caudomedial position of temporal axons in the contralateral optic tract (Fig. 5c). Examining the ipsilateral hemispheres of each of these topographically labeled samples reveals that ipsilateral axons are appropriately positioned within the topographic map in the optic tract. Specifically, ventral RGC axons are situated in the rostrolateral aspect and temporal RGC axons in the caudomedial aspect of the ipsilateral optic tract. However, as in Fig. 3, it is apparent that ipsilateral RGC axons, though grossly in register with the topographic map, are situated slightly more laterally than their contralateral counterparts (see Fig. 5d for summary; ipsilateral RGC axons are shown as checkered areas in the lateral aspect of the tract to compare with contralateral RGC axons position, in solid colors). 


\section{Ipsilateral RGC neurites self-fasciculate more than contralateral RGC neurites}

Having demonstrated that ipsilateral and contralateral RGC axons are consistently segregated in the optic nerve and tract, and that such eye-specific axon organization occurs in addition to topographic organization, we next sought to identify a mechanism by which ipsilateral and contralateral axons are organized. Based in part on qualitative observations from prior work in our lab (L.-C. Wang, Dani, Godement, Marcus, \& Mason, 1995), as well as the appearance of changes in ipsilateral axon bundling in vivo (Fig. 1c-e), we aimed to determine if ipsilateral RGC axons fasciculate more than contralateral axons. Fasciculation, the bundling together of axons, is an important mechanism in axon guidance and circuit formation, as nearly all axons grow along other axons as they extend towards their targets (Raper \& Mason, 2010; L. Wang \& Marquardt, 2013). Evidence from the olfactory and visual systems indicates that axon-axon interactions are critical for the formation of neural circuits (Ebrahimi \& Chess, 2000; Imai \& Sakano, 2011; Weth, Fiederling, Gebhardt, \& Bastmeyer, 2014). Thus, we hypothesized that ipsilateral and contralateral RGC axons may differentially self-fasciculate, which could in turn contribute to their segregation from one another in the optic nerve and tract.

We tested this hypothesis using an in vitro retinal explant system to analyze neurite bundle widths as a measure of degree of fasciculation (Fig. 6a). Explants were taken from ventrotemporal (VT) or dorsotemporal (DT) retinal quadrants at E14.5, when VT retina predominantly, if not exclusively, produces ipsilateral RGCs, and DT retina is completely devoid of ipsilateral RGCs (Colello \& Guillery, 1990). Thus, VT explants represent ipsilateral RGCs and DT explants represent contralateral RGCs. After being cultured on a growth-permissive laminin substrate for 24 hours (h), explants were fixed, immunostained for neurofilament (NF) to visualize neurites (Fig. 6b), and analyzed. In the micrographs, two rings were digitally placed 250 and $500 \mu \mathrm{m}$ away from the perimeter of the explant body, and neurite bundle (or fascicle) widths were measured along each ring (Fig. 6a).

When grown on laminin alone, VT neurites form thicker fascicles than DT neurites (light green and light magenta, respectively, Fig. 6c-d). This is true at both ring radii (Fig. 6c, at $250 \mu \mathrm{m}, \mathrm{p}=3.85 \times 10^{-22}$; Fig. $6 \mathrm{~d}$, at $500 \mu \mathrm{m}, \mathrm{p}=1.29 \times 10^{-42}$, Mann Whitney $U$ test $\mathrm{n}=$ 18 VT explants, 17 DT explants, from 4 independent experiments). Additionally, fascicles are thicker at the $500 \mu \mathrm{m}$ radius compared with the $250 \mu \mathrm{m}$ ring for both explant types (comparison not shown graphically; VT $250 \mu \mathrm{m}$ versus $500 \mu \mathrm{m}, \mathrm{p}=8.95 \times 10^{-33}$; DT 250 $\mu \mathrm{m}$ versus $500 \mu \mathrm{m}, \mathrm{p}=2.63 \times 10^{-16}$, Mann Whitney $U$ test).

We next tested if the intrinsic difference in fasciculation behaviors between ipsilateral (VT) and contralateral (DT) neurites would be affected by the presence of extrinsic cues from the chiasm cells in the culture system. Exposure to chiasm cues in vitro would better recapitulate axons growing in the optic tract, where RGC axons have navigated cues at the midline to project ipsi- or contralaterally into the optic tract. The optic chiasm is repulsive to ipsilateral RGC axons in vivo, but contralateral axons are able to navigate through the chiasm midline. Therefore, we hypothesized that the presence of dissociated chiasm cells would preferentially increase fasciculation of ipsilateral (VT) neurites compared to contralateral (DT) neurites. Because dissociated chiasm cells are generally inhibitory to RGC neurite outgrowth (L.-C. Wang et al., 1995), we plated dissociated chiasm cells at a 
low density in the co-culture system $\left(3.5 \times 10^{5}\right.$ cells $\left./ \mathrm{ml}\right)$, so that there was sufficient neurite outgrowth to analyze fasciculation. After growing retinal explants on laminin with chiasm cells for $24 \mathrm{~h}$ (Fig. 6b, right panels), we again measured neurite bundle widths along rings placed $250 \mu \mathrm{m}$ and $500 \mu \mathrm{m}$ away from the perimeter of the explant body. The intrinsic preference of VT neurites to fasciculate more than DT neurites persisted in the presence of extrinsic cues from dissociated chiasm cells (dark green and dark magenta lines, respectively, Fig. 6e, at $250 \mu \mathrm{m}, \mathrm{p}=3.23 \times 10^{-4}$; Fig. 6f, at $500 \mu \mathrm{m}, \mathrm{p}=4.46 \times 10^{-8}$, Mann Whitney $U$ test; $\mathrm{n}=16 \mathrm{VT}$ explants, $16 \mathrm{DT}$ explants, from 3 independent experiments). Similar to the laminin-only condition, DT neurites grown with chiasm cells formed thicker fascicles at the $500 \mu \mathrm{m}$ radius compared with the $250 \mu \mathrm{m}$ radius (comparison not shown graphically; $\mathrm{p}=0.04$, Mann Whitney $U$ test). VT neurites grown with chiasm cells, however, did not differ in fascicle width between the two radii (comparison not shown graphically; $\mathrm{p}=$ 0.53 , Mann Whitney $U$ test). This may be due to the fact that chiasm cells are more growth refractory to VT neurites than DT neurites, and VT neurites were shorter overall when cultured with chiasm cells, resulting in fewer neurite bundles crossing the $500 \mu \mathrm{m}$ ring.

While we expected the addition of chiasm cells to the culture system to preferentially increase fasciculation of VT neurites, we found that both DT and VT neurites formed thicker bundles in the chiasm co-culture than they did when grown on laminin alone (Fig. 6e-f). VT neurites form thicker fascicles at both radii when grown with chiasm cells than when grown only on laminin (dark and light green lines, respectively, Fig. 6e, at $250 \mu \mathrm{m}, \mathrm{p}=7.24 \times$ $10^{-18}$; Fig. 6f, at $500 \mu \mathrm{m}, \mathrm{p}=0.03$, Mann Whitney $U$ test). DT neurites grown with chiasm cells form thicker bundles than chiasm-naïve DT neurites at the $250 \mu \mathrm{m}$ radius (dark and light magenta lines, respectively, Fig. $6 e, p=6.76 \times 10^{-21}$, Mann Whitney $U$ test), but not at the $500 \mu \mathrm{m}$ radius (dark and light magenta lines, respectively, Fig. 6f, respectively, $\mathrm{p}=0.11$, Mann Whitney $U$ test). The lack of difference for DT neurites with and without chiasm cells at the $500 \mu \mathrm{m}$ radius could indicate that the addition of chiasm cells has less of an effect on DT neurites compared to VT neurites, which formed thicker bundles at both radii in the chiasm co-culture condition. In sum, VT (ipsilateral) neurites have an intrinsic preference to fasciculate into larger bundles than DT (contralateral) neurites, and extrinsic cues from dissociated chiasm cells lead to increased self-fasciculation in both populations, though perhaps more so in VT explants.

Theoretically, the presence of more neurites could increase the likelihood of fasciculation in culture. Therefore, we performed a series of additional analyses to assess first, whether VT and DT explants have comparable neurite outgrowth in these culture conditions, and second, if neurite outgrowth affects neurite fasciculation. To address the first issue, we examined both explant size and total neurite outgrowth of VT and DT explants. Explant size was kept consistent in the experiment, with samples deviating from an explant body radius of $150 \mu \mathrm{m}$ by $\pm 50 \mu \mathrm{m}$ excluded from analysis. There were no differences in explant radius between VT and DT explants, nor between growth condition (i.e., with or without chiasm cells, dark and light boxes, respectively, Fig. 6e; whiskers indicate minimum and maximum values). However, even while holding explant size consistent, there may be differences in neurite outgrowth between VT and DT explants. In order to assess the total amount of neurite outgrowth, widths of neurite bundles intersecting the $250 \mu \mathrm{m}$ radius ring were summed for each explant. Based on this measure of summed bundle widths, VT explants have 
significantly more outgrowth than DT explants when grown on laminin (Fig. 6f, mean \pm

SEM: $\mathrm{VT}=157.7 \mu \mathrm{m} \pm 13.4, \mathrm{DT}=107.5 \mu \mathrm{m} \pm 11.5, \mathrm{p}=0.0073$, One-Way ANOVA), even though the explants were no different in size (Fig. 6e). Co-culturing explants with dissociated chiasm cells had the expected growth-refractory effect on neurite outgrowth, with both explant types grown on chiasm cells having stunted outgrowth compared to those grown on laminin only (Fig. 6f, VT vs VT+OC, $\mathrm{p}<0.0001$; DT vs DT+OC, $\mathrm{p}=0.002$, OneWay ANOVA). There was, however, no significant difference in neurite outgrowth between $\mathrm{VT}$ and DT explants that were co-cultured with chiasm cells (Fig. 6f, mean \pm SEM: VT+OC $=66.6 \mu \mathrm{m} \pm 7.2, \mathrm{DT}+\mathrm{OC}=48.3 \mu \mathrm{m} \pm 7.5, \mathrm{p}=0.44$, One-Way ANOVA).

Because VT explants grown on laminin have greater neurite outgrowth (i.e., summed bundle widths) than DT explants, we examined whether the greater self-fasciculation found in VT explants might be simply a result of increased neurite number. Therefore, a subset of VT and DT explants that were matched for total neurite outgrowth was re-analyzed to determine if increased VT neurite fasciculation is a distinct phenomenon from increased VT neurite outgrowth. Five VT explants and six DT explants (from 3 experiments) with total neurite outgrowth restricted to $90-150 \mu \mathrm{m}$ (around the $250 \mu \mathrm{m}$ radius ring) were analyzed; - there was no statistical difference in neurite outgrowth between VT and DT explants within this subset, so we therefore considered them equivalent in total neurite outgrowth. All of the differences in neurite fasciculation identified in the full data set were still present, although slightly smaller in magnitude, with VT neurites forming thicker bundles than DT neurites at both radii (Fig. 6g; VT vs DT, p = 0.0017, $250 \mu \mathrm{m}$ ring; $\mathrm{p}=1.64 \times 10^{-14}, 500 \mu \mathrm{m}$ ring; 250 $\mu \mathrm{m}$ vs $500 \mu \mathrm{m}$ ring, $\mathrm{p}=4.55 \times 10^{-16}$, VT neurites; $\mathrm{p}=1.86 \times 10^{-7}$, DT neurites; Mann Whitney $U$ test). Thus, VT neurites both grow and self-fasciculate more than DT neurites in these culture conditions, and the self-fasciculation behavior is a phenomenon distinct from increased neurite outgrowth.

\section{Ipsilateral axon fasciculation and organization are maintained in the EphB1 ${ }^{-/-}$optic tract}

We next interrogated the relationship between axon self-association in the optic tract and targeting in the dLGN by examining the EphB $1^{-/-}$mutant mouse. EphB1 is expressed by ipsilateral RGCs and mediates ipsilateral axon divergence at the optic chiasm via repulsion from midline ephrin-B2. Loss of EphB1 leads to a reduction, but not complete loss of the ipsilateral RGC axon projection (Williams et al., 2003). Instead of turning away from the optic chiasm, a subset of ipsilateral RGC axons in the EphB1 $1^{-1-}$ aberrantly crosses the midline and mis-projects to the contralateral hemisphere. However, despite this erroneous decussation choice, aberrantly crossed EphB1 ${ }^{-/-}$axons target the ipsilateral zone of the opposite dLGN, rather than the dorsal tip of the dLGN, where normal contralateral axons from VT retina target (Rebsam, Petros, \& Mason, 2009). Thus, the EphB1 ${ }^{-1-}$ mutant provides a model in which a subset of ipsilateral RGC axons misroute at the optic chiasm, but grossly target normally in the dLGN (Fig. 7a), allowing us to assess the relationship between tract organization and targeting.

We first asked whether loss of EphB1 perturbs the overall eye-specific organization of RGC axons in the optic tract. Using whole-eye DiI and DiD anterograde labeling as before (Fig. 7b), we examined the eye-specific axon organization in frontal (Fig. 7c) and horizontal 
sections (Fig. 7d) of P0 wild-type and EphB1 ${ }^{-/-}$mutant mice. In both planes of view, it is apparent that the remaining ipsilateral axons retain their normal position in the lateral optic tract, segregated from contralateral axons. However, the ipsilateral axons appear less fasciculated compared to those in the wild-type tract. Thus, loss of EphB1 does not affect positioning of ipsilateral and contralateral axons in the optic tract, but may be involved in coherence of the ipsilateral fibers in the pathway.

The most intriguing question in the EphB1 $1^{-/-}$mutant is where in the optic tract the aberrantly crossed axons are positioned (Fig. 7e). Answering this question could provide clues to the relationship between tract organization and targeting in the retinogeniculate system. If aberrantly crossed axons associate with normal contralateral axons in the optic tract (Fig. $7 \mathrm{e}^{1}$ ), then bundling partners in the tract would arguably be inconsequential to initial targeting decisions, given that aberrantly crossed fibers still target the ipsilateral zone in the dLGN (Fig. 7a, right). On the other hand, finding that aberrantly crossed axons retain an association with the remaining ipsilateral axons in the contralateral hemisphere (Fig. $7 \mathrm{e}^{2}$ ) would support a model in which maintenance of cohort-specific association in the optic tract is related to targeting.

To determine if aberrantly crossed $\mathrm{EphB} 1^{-/-}$axons associate with ipsilateral or contralateral axons, we crossed the SERT-Cre::zsGreen reporter mouse, labeling ipsilateral RGCs, with the EphB1 ${ }^{-/-}$mutant to produce SERT-Cre::zsGreen;EphB1 (SERT;EphB1) offspring. EphB1 and SERT are both downstream of the ipsilateral-specific transcription factor, Zic2, but in independent transcriptional pathways (Garcia-Frigola, Carreres, Vegar, Mason, \& Herrera, 2008; Garcia-Frigola \& Herrera, 2010). Thus, in EphB1 ${ }^{-/}$mutants, the remaining ipsilateral axons as well as aberrantly crossed axons should both express SERT. To confirm that loss of EphB1 does not affect SERT expression in RGCs, we compared numbers of zsGreen+ cells in flat-mounted retinae from SERT;EphB1 ${ }^{+/+}$, SERT;EphB $1^{+/-}$, and SERT;EphB1 ${ }^{-/-}$animals at P0 (Fig. 7f-g). There was no statistically significant difference in zsGreen + cell counts between genotypes (mean \pm SEM: SERT;EphB1 $1^{+/+}=2703 \pm 315.4, \mathrm{n}$ $=7 ;$ SERT;EphB $1^{+/-}=1896 \pm 403, \mathrm{n}=4 ;$ SERT;EphB1 ${ }^{-/-}=2404 \pm 203.7, \mathrm{n}=6 ; \mathrm{p}=0.24$, One-Way ANOVA). Therefore, we concluded that the SERT+ population of RGCs in the EphB1 $1^{-/}$mutant indeed contains both the remaining ipsilateral RGCs and those that aberrantly send their axons contralaterally at the optic chiasm.

We next examined the arrangement of zsGreen+ axons (green brackets) in the optic tracts (labeled with NF, magenta brackets) of SERT;EphB1 ${ }^{+/+}$and SERT;EphB1 $1^{-/}$animals at P0 (Fig. 7h). If aberrantly crossed axons associate with normal contralateral axons (as in Fig. $7 \mathrm{e}^{1}$ ), then the zsGreen+ region of the tract would expand medially, with true ipsilateral axons in the lateral tract and aberrantly crossed axons straying into the medial tract with contralateral axons. If, on the other hand, aberrantly crossed axons retain an association with the remaining ipsilateral axons (as in Fig. $7 \mathrm{e}^{2}$ ), then we would expect no difference in the span of zsGreen+ axons in the tract, with both true ipsilateral and aberrantly crossed axons positioned in the lateral aspect of the optic tract. Consistent with the latter hypothesis, there were no differences in the total tract width (Fig. 7i; $p=0.91$, One-Way ANOVA), or in the percentage of the tract occupied by zsGreen+ axons (Fig. 7j; $p=0.67$, One-Way ANOVA). These findings indicate that despite incorrectly decussating, aberrantly crossed VT RGC 
axons retain an association with axons of the same cohort, i.e., ipsilateral RGCs, but on the wrong side of the brain.

To address whether loss of EphB1 affects ipsilateral self-fasciculation, we examined neurite bundle widths in vitro from EphB1 ${ }^{-/-}$DT and EphB1 ${ }^{-/-}$VT explants grown for $24 \mathrm{~h}$ on laminin (Fig. 7k). As with wild-type explants, explant size was kept consistent, with no difference between EphB1 ${ }^{-/-}$VT and EphB1 $1^{-/-}$DT explant body radius (Fig. 71, p = 0.99, One-Way ANOVA). Again, however, there was a significant difference in neurite outgrowth measured by summing bundle widths around the $250 \mu \mathrm{m}$ ring, with EphB1 $1^{-/-} \mathrm{VT}$ explants extending more neurites than EphB1 ${ }^{-/-}$DT explants (Fig. 7m; mean \pm SEM: VT $=201.4 \mu \mathrm{m}$ $\pm 15.1, \mathrm{n}=16$; DT $=160.5 \mu \mathrm{m} \pm 8.4, \mathrm{n}=19 ; \mathrm{p}=0.037$, One-Way ANOVA). Therefore, in addition to analyzing the full set of EphB1 $1^{-/-}$VT and EphB1 $1^{-/}$DT explants (Fig. 7n), we also assessed a subset of explants $\left(n=7\right.$ explants, EphB1 ${ }^{-/-}$VT; $n=11$ explants, EphB1 $1^{-/-}$ DT; from 3 experiments) matched for neurite outgrowth (Fig. 7o), as was done in the wildtype in vitro experiments (Fig. 6g). In both the full data set and the outgrowth-matched subset, EphB1 ${ }^{-/-}$VT neurites self-fasciculate more than EphB1 $1^{-/-}$DT neurites when grown on laminin (Fig. $7 \mathrm{n}$, full data set, $\mathrm{p}=1.20 \times 10^{-7}, 250 \mu \mathrm{m}$ ring; $\mathrm{p}=2.74 \times 10^{-14}, 500 \mu \mathrm{m}$ ring; Fig. 7o, outgrowth-matched subset, $p=2.60 \times 10^{-4}, 250 \mu \mathrm{m}$ ring; $\mathrm{p}=6.25 \times 10^{-8}, 500$ $\mu \mathrm{m}$ ring; Mann Whitney $U$ test). EphB1 $1^{-l-} \mathrm{DT}$ and EphB1 $1^{-/-} \mathrm{VT}$ neurites form slightly thicker bundles at $500 \mu \mathrm{m}$ from the explant body than they do at $250 \mu \mathrm{m}$ away (Fig. 7n, full data set, $\mathrm{p}=1.44 \times 10^{-6}$, EphB1 $1^{-/-} \mathrm{VT} ; \mathrm{p}=0.0043$, EphB1 $1^{-/-} \mathrm{DT}$; Fig. 7o, outgrowthmatched subset, $\mathrm{p}=1.08 \times 10^{-4}, \mathrm{EphB} 1^{-/-} \mathrm{VT} ; \mathrm{p}=0.0012$, EphB1 $1^{-/-} \mathrm{DT}$; Mann Whitney $U$ test).

In sum, loss of EphB1 does not eliminate the intrinsic preference of ipsilateral neurites to fasciculate more than contralateral neurites in vitro, nor does it affect fasciculation dynamics within either explant type. In combination with the in vivo data presented above from the SERT;EphB1 optic tract, we conclude that the appearance of mild defasciculation of ipsilateral axons in the optic tract of EphB1-/- mutants in Fig. 7c-d is not a reflection of decreased fasciculation, but rather of the decreased number of ipsilaterally projecting axons. The apparent gaps between remaining ipsilateral axons in Fig. 7c-d are likely occupied by aberrantly crossed axons, as reflected by zsGreen+ signal in Fig. 7h, and thus coherence of the ipsilateral cohort is maintained.

\section{Discussion}

There is a growing appreciation that axons are organized by topographic, typographic, and/or chronotopic principles in tracts leading to their targets (e.g., Kashima, Rubel, \& Seidl, 2013; Miller et al., 2010) and that pre-target axon organization may be a crucial step in circuit formation (Imai et al., 2009; Zhou et al., 2013). However, particularly in the visual system, the extent of and mechanisms contributing to pre-target axon organization remain unclear. Here we provided the first direct comparison of two modes of pre-target axon organization in the retinogeniculate pathway, showing that RGC axons are organized by both laterality (a form of typography) and topography in the mouse optic nerve and tract, and that the eye-specific (typographic) map is shifted slightly laterally to the topographic map in the tract. Such detailed analyses, which incorporate multiple modes of axon order, are essential 
for investigating mechanisms of pre-target axon organization and determining if tract order is necessary for appropriate targeting.

We probed selective fasciculation and coherence of axon cohorts as mechanisms of pretarget axon organization. Ipsilateral neurites fasciculate more than contralateral neurites in vitro, suggesting that selective fasciculation may be one mechanism contributing to segregation of ipsilateral and contralateral RGC axons in the optic nerve and tract. Ipsilateral (VT) neurites also grow more exuberantly than contralateral (DT) neurites in vitro, but this is a distinct, if not independent, behavior from their enhanced fasciculation. Further, in the EphB1 ${ }^{-1-}$ mutant optic tract, aberrantly crossed axons associate with ipsilateral axons, with which they target in the dLGN (Rebsam et al., 2009). This finding suggests that cohortspecific axon association along the pathway may be related to targeting.

\section{Multiple modes of RGC axon organization in the mouse retinogeniculate pathway}

In agreement with previous reports (Godement et al., 1984), we found that ipsilateral axons course within the lateral optic tract. In addition to showing that this position is consistent throughout development, we also demonstrate that ipsilateral and contralateral axons are well segregated, with the majority of contralateral axons avoiding the lateral optic tract. It is important to note that the word "segregation" is not meant to indicate a binary or complete divergence of the ipsilateral and contralateral RGC axon cohorts. Indeed, it is clear in the high resolution of the wholemounted tract (Fig. 2e-f and Movies 1-2) that individual ipsilateral and contralateral axons are closely apposed to one another in some areas of the tract. Rather, ipsilateral and contralateral axons are organized along the continuum of the lateral-medial axis of the optic tract, and are thus segregated on a population level.

Genetic labeling of ipsilateral RGCs shows that ipsilateral axons are pre-sorted in the optic nerve prior to navigating the optic chiasm. Thus, while ipsilateral and contralateral cohorts of RGC axons intermingle in and around the chiasm, there is clear organization and segregation between the two populations in the proximal optic nerve and throughout the optic tract. As is the case with topographic order of RGC axons (Chan \& Guillery, 1994), ipsilateral RGC axons become progressively less well sorted in the optic nerve as they near the chiasm, and re-establish their order after entering the tract, suggestive of active organizational mechanisms at play in the optic tract.

RGC axons are also arranged topographically in the optic nerve and tract, with the dorsoventral axis of the retina mapped to the medial-lateral axis in the tract, and the nasaltemporal axis mapped to the rostrocaudal axis of the tract (summarized in Fig. 5d). Previous studies reported little organization between nasal and temporal RGC axons in the optic tract (Chan \& Guillery, 1994; Plas et al., 2005; Reese \& Baker, 1993). Our results instead indicate a coarse rostromedial to caudolateral organization of nasal and temporal axons. Indeed, nasal and temporal RGC axons even preserve their segregation through the chiasm (Fig. $4 \mathrm{c}^{2}$ ). Previous reports used unconventional planes of section (i.e., neither standard frontal or horizontal planes), and as such, the organization along the rostrocaudal axis may not have been readily appreciated. Our observations agree with earlier conclusions of dorsal and ventral RGC axon position in the medial and lateral optic tract, respectively (Chan \& Chung, 1999; Chan \& Guillery, 1994; Plas et al., 2005; Reese \& Baker, 1993; Reh et al., 1983; 
Torrealba et al., 1982). We expand upon these earlier reports by also identifying a slight rostrocaudal segregation between ventral and dorsal axons in the tract and showing that the two cohorts overlap in the optic chiasm as they switch orientation from the nerve into the tract.

In the first direct comparison of two distinct modes of axon organization in a developing axon tract, we found that the eye-specific map is largely in register with the topographic map in the optic tract, although ipsilateral RGC axons are situated laterally relative to the contralateral topographic map. This hints at a possible hierarchy among different organizational modes. Given that binocular vision is a later evolutionary development (Petros, Rebsam, \& Mason, 2008), it is conceivable that eye-specific organization between ipsilateral and contralateral axons was superimposed onto the existing topographic order, which would explain the slight shift laterally of ipsilateral axons relative to the topographic map. This would, in an evolutionary sense, align with the principal of chronotopic order, in which younger axons are added to the lateral edge of the optic tract, with older axons pushed medially. However, in the strict sense of chronotopy (as opposed to an evolutionary-agebased order), eye-specific axon organization may be at odds with chronotopic organization in the optic tract. Age-related order of RGC axons has been described (Walsh \& Guillery, 1985), but because late-born contralateral axons from VT retina are situated more medially than ipsilateral axons, chronotopic order may be subordinate to eye-specific organization, and potentially also to topographic order. Given the diversity of RGC subtypes, RGC axons may also be typographically organized based on functional and transcriptional identity. Genetic dissection of RGC subtypes (e.g., Martersteck et al., 2017) will allow further exploration of typographic organization of axons in the visual system and identification of any correspondence between pre-target axon organization and targeting.

\section{Selective fasciculation of ipsilateral RGC axons}

Ipsilateral axon self-association decreases in the proximal optic nerve near the chiasm but is reestablished and maintained in the optic tract (Fig. 1c-e). Progressive unraveling of ipsilateral axons nearing the chiasm was described previously, based on retrograde labeling from the optic tract (Colello \& Guillery, 1990). Given this apparent dynamic fasciculation of ipsilateral axons in static images from SERT-Cre::zsGreen brains, we tested whether ipsilateral and contralateral axons have different fasciculation behaviors. Neurites from VT retinal explants (predominantly ipsilateral RGCs) self-fasciculate more than neurites from DT explants (contralateral RGCs). While small in magnitude, this intrinsic difference in selffasciculation may contribute to establishment and maintenance of eye-specific axon segregation in the optic tract.

However, we also found that VT explants extend more neurites than DT explants, even when controlling for explant size. Analysis of outgrowth-matched VT and DT explants showed that the increase in VT neurite self-fasciculation is not simply a byproduct of increased neurite outgrowth in VT explants, though the two are likely related. While neurite outgrowth could affect the extent of fasciculation, fasciculation fosters increased axon extension rates (Bak \& Fraser, 2003; Bruce, Brown, Smith, Fuerst, \& Erskine, 2017). Thus, VT explants may extend more neurites in vitro in part because VT neurites fasciculate more than DT 
neurites. However, ipsilateral RGCs may also have an inherent proclivity for robust neurite outgrowth in vitro. Transcriptional profiling and neurogenesis studies of ipsilateral and contralateral RGCs suggests that ipsilateral RGCs possess a more immature transcriptional profile compared with contralateral RGCs (Marcucci et al., 2016; Q. Wang, Marcucci, Cerullo, \& Mason, 2016), which could contribute to enhanced VT neurite outgrowth in vitro.

Extrinsic cues from the optic chiasm encourage greater fasciculation of both VT and DT neurites in vitro, but the intrinsic difference in fasciculation behavior is maintained. In vivo, there are likely additional cues within and along the optic tract, as yet unidentified, which also contribute to the organization of ipsilateral and contralateral axons. The in vitro assay of self-fasciculation presented here can be used in the future to assess putative fasciculation molecules that are differentially expressed between ipsi- and contralateral RGCs, such as Semaphorins (Q. Wang et al., 2016), which are important players in pre-target axon organization in the corpus callosum and olfactory nerve (Imai et al., 2009; Zhou et al., 2013).

In the in vitro bundle width assay, the larger fascicles along the $500 \mu \mathrm{m}$ radius compared to the $250 \mu \mathrm{m}$ radius suggest that the longer neurites extend near each other, the more they are disposed to fasciculate with one another. Alternatively, neurites are less densely packed the farther they extend from the explant, so the increased fasciculation at $500 \mu \mathrm{m}$ may instead reflect the preference to grow along other neurites rather than substrate. Live imaging experiments could reveal more details regarding selective fasciculation dynamics, and the extent to which fasciculation occurs only at the growth cone or also along axon shafts (Smit, Fouquet, Pincet, Zapotocky, \& Trembleau, 2017). Future studies using live imaging will reveal more details of differential fasciculation dynamics between ipsilateral and contralateral RGC axons.

Indeed, live imaging and other variations on our in vitro assay could elucidate additional important details of retinal axon behavior, and, in particular, could provide more insight into how axons use fasciculation to segregate from one another. For instance, one shortcoming of the assay as presented here is that by only comparing VT and DT explants, we cannot rule out the possibility that the differences in neurite bundle width between the two explant types is due to topographic differences, rather than ipsilateral contralateral identity. Previous comparisons of retinal quadrants found little to no difference in neurite outgrowth and behavior between DT, dorsonasal (DN), and ventronasal (VN) neurites in vitro (Erskine et al., 2000; L.-C. Wang et al., 1995; L.-C. Wang, Rachel, Marcus, \& Mason, 1996), but it would be informative nonetheless to ascertain more conclusively whether topographic origin affects neurite self-fasciculation behavior. Even more compelling would be an in vitro assay that provided neurites from different retinal regions choices of fasciculation partners from like and unlike neurites. Such an assay could theoretically be used to determine whether topographic identity or ipsilateral/contralateral identity is a stronger force in driving the choice of fasciculation partners, and time-lapse imaging in such a paradigm could unveil novel and important dynamics of developing retinal axon behavior. 


\section{Maintaining axon cohort coherence along the pathway}

To further probe ipsilateral axon self-association as a mechanism of eye-specific axon organization in the tract, and relate it to targeting, we examined RGC axon organization and fasciculation in the EphB1 ${ }^{-/}$mutant. Given the dearth of knowledge of molecular profiles of RGC axons as they navigate the optic tract and the molecular environment within the tract, the EphB1 $1^{-/-}$mutant provided a tractable way to relate tract organization, specifically ipsilateral axon self-association, and targeting in vivo. In the EphB1 $1^{-/}$mutant, a subset of ipsilateral RGC axons aberrantly crosses the midline and projects to the contralateral hemisphere, but targets the ipsilateral zone in the opposite dLGN (Rebsam et al., 2009; Williams et al., 2003). Loss of EphB1 did not perturb the position of remaining ipsilateral RGC axons in the tract, but these axons appeared slightly de-fasciculated. In vitro, however, VT EphB1 $1^{-/}$neurites still formed thicker fascicles than DT EphB1 $1^{-/}$neurites, demonstrating that EphB1 is not necessary for ipsilateral RGC axon fasciculation.

A qualitative comparison of the differences in self-fasciculation between VT and DT neurites from wild-type (Fig. 6c, d, and g) and EphB1 ${ }^{-/-}$(Fig. 7n and o) explants hints that the magnitude of the difference in neurite bundle widths is smaller in the EphB1 ${ }^{-/-}$mutants than wild-type. Because these animals were bred on different genetic backgrounds, we could not directly quantify such a difference, but it leaves open the possibility that, while not necessary, EphB1 may still be involved in ipsilateral axon self-fasciculation. Future studies could directly test such a role by inhibiting EphB1 function in vitro in wild-type explants and assessing whether there is in fact a decrease in self-fasciculation in VT neurites.

However, our in vivo data support the interpretation that EphB1 is not necessary for ipsilateral axon coherence along the optic tract, as zsGreen labeling in the SERT;EphB1 $1^{-1-}$ optic tract, which labels remaining ipsilateral axons and aberrantly crossed axons (both originiating from VT retina), showed that both groups bundle together in the lateral optic tract. This finding provides insight into the relationship between tract order and targeting. If axon organization is relevant to targeting, one would predict, as we show, that aberrantly crossed axons from VT retina in the EphB1 $1^{-/-}$mutant maintain an association with the remaining ipsilateral axons (from the other eye), and course together to the ipsilateral zone in the dLGN. We propose that this is due in part to maintenance of ipsilateral axon selfassociation, which could occur cell-autonomously via the preservation of ipsilateral RGC specific fasciculation cues.

The association of aberrantly crossed axons with the rest of the ipsilateral RGC axon cohort corresponds to their targeting of the ipsilateral zone in the dLGN. However, we cannot yet show directly that axon organization in the tract influences targeting. In order to directly test that hypothesis, more information is needed on ipsilateral-specific fasciculation factors, extrinsic cues in the tract, and the dynamics of molecular expression in growth cones and axon shafts of ipsilateral and contralateral RGCs at different points within the tract. Growth cones navigating towards, through, and away from midline choice points adjust their receptor expression to continue their journey to the target (e.g., Dodd, Morton, Karagogeos, Yamamoto, \& Jessell, 1988), but guidance molecule expression patterns on RGC axons postchiasm are largely unknown. Expression of guidance molecules by RGC axons within the optic tract may be regulated by local translation (Hornberg, Cioni, Harris, \& Holt, 2016), so 
future experiments testing the involvement of axon order in the optic tract on targeting in the dLGN will need spatiotemporal precision in manipulations of RGC axons. That is, it will be key to selectively affect axons within the optic tract, and not those prior to the chiasm, so as to differentiate between effects on decussation versus effects on axon organization in the tract. The continuous wave of RGC axon outgrowth from the retina during late embryogenesis makes this particularly challenging, as does the fact that many guidance cues act at multiple points along the pathway.

Distinguishing between the effects of aberrant midline choice versus aberrant tract order on targeting is necessary, because each developmental step of the pathway may prime axons for successful navigation of the subsequent step. Evidence from the auditory system indicates that correct midline choice primes axons for appropriate synapse maturation in their target (Michalski et al., 2013), and in the thalamocortical system, correct axon organization in the tract through an intermediate target is necessary for distinct topographic mapping of thalamic axons into the cortex (Dufour et al., 2003; Garel, Yun, Grosschedl, \& Rubenstein, 2002; Seibt et al., 2003). Thus, it is possible that appropriate organization of axons in the optic tract is a prerequisite for correct early targeting choices. Expanding our understanding of selective fasciculation and axon-axon interactions along the full extent of a circuit will be crucial to understanding how neural circuits are built during development.

\section{Supplementary Material}

Refer to Web version on PubMed Central for supplementary material.

\section{Acknowledgments}

This work was supported by NIH Grants R01 EY012736, T32 EY013933, and Vision Core Grant 5P30 EY019007. Confocal imaging was performed at the Confocal and Specialized Microscopy Shared Resource of the Herbert Irving Comprehensive Cancer Center at Columbia University, supported by NIH grant P30 CA013696 (National Cancer Institute), under supervision of Drs. T. Swayne and L. Pon. R. Blazeski and H. Melikyan provided technical assistance, E. Dodd performed pilot experiments on the in vitro assay, and N. Gallerani assisted with an early version of the bundle width assay. Dr. J. Bylund wrote python code for statistical analyses. We thank members of the Mason and Dodd Labs, particularly Dr. Jane Dodd, Dr. Felix Fiederling, and Melissa Lee, for critical reading of the manuscript and feedback on experimental analysis. We also thank Drs. Wes Grueber and Ulrich Hengst for thoughtful comments and feedback throughout the study.

\section{References}

Bak M, Fraser SE. Axon fasciculation and differences in midline kinetics between pioneer and follower axons within commissural fascicles. Development. 2003; 130(20):4999-5008. DOI: 10.1242/dev.00713 [PubMed: 12952902]

Bruce FM, Brown S, Smith JN, Fuerst PG, Erskine L. DSCAM promotes axon fasciculation and growth in the developing optic pathway. Proc Natl Acad Sci U S A. 2017; 114(7):1702-1707. DOI: 10.1073/pnas.1618606114 [PubMed: 28137836]

Chan SO, Chung KY. Changes in axon arrangement in the retinofugal [correction of retinofungal] pathway of mouse embryos: confocal microscopy study using single- and double-dye label. J Comp Neurol. 1999; 406(2):251-262. [PubMed: 10096609]

Chan SO, Guillery RW. Changes in fiber order in the optic nerve and tract of rat embryos. J Comp Neurol. 1994; 344(1):20-32. DOI: 10.1002/cne.903440103 [PubMed: 8063954]

Colello RJ, Guillery RW. The early development of retinal ganglion cells with uncrossed axons in the mouse: retinal position and axonal course. Development. 1990; 108(3):515-523. [PubMed: 2340812] 
Dodd J, Morton SB, Karagogeos D, Yamamoto M, Jessell TM. Spatial regulation of axonal glycoprotein expression on subsets of embryonic spinal neurons. Neuron. 1988; 1(2):105-116. [PubMed: 3272160]

Drager UC. Birth dates of retinal ganglion cells giving rise to the crossed and uncrossed optic projections in the mouse. Proc R Soc Lond B Biol Sci. 1985; 224(1234):57-77. [PubMed: 2581263]

Dufour A, Seibt J, Passante L, Depaepe V, Ciossek T, Frisen J, Vanderhaeghen P. Area specificity and topography of thalamocortical projections are controlled by ephrin/Eph genes. Neuron. 2003; 39(3): 453-465. [PubMed: 12895420]

Ebrahimi FA, Chess A. Olfactory neurons are interdependent in maintaining axonal projections. Curr Biol. 2000; 10(4):219-222. [PubMed: 10704414]

Erskine L, Williams SE, Brose K, Kidd T, Rachel RA, Goodman CS, Mason CA. Retinal ganglion cell axon guidance in the mouse optic chiasm: expression and function of robos and slits. J Neurosci. 2000; 20(13):4975-4982. [PubMed: 10864955]

Garcia-Frigola C, Carreres MI, Vegar C, Mason C, Herrera E. Zic2 promotes axonal divergence at the optic chiasm midline by EphB1-dependent and -independent mechanisms. Development. 2008; 135(10):1833-1841. DOI: 10.1242/dev.020693 [PubMed: 18417618]

Garcia-Frigola C, Herrera E. Zic2 regulates the expression of Sert to modulate eye-specific refinement at the visual targets. EMBO J. 2010; 29(18):3170-3183. DOI: 10.1038/emboj.2010.172 [PubMed: 20676059]

Garel S, Yun K, Grosschedl R, Rubenstein JL. The early topography of thalamocortical projections is shifted in Ebf1 and Dlx1/2 mutant mice. Development. 2002; 129(24):5621-5634. [PubMed: 12421703]

Godement P, Salaun J, Imbert M. Prenatal and postnatal development of retinogeniculate and retinocollicular projections in the mouse. J Comp Neurol. 1984; 230(4):552-575. DOI: 10.1002/ cne.902300406 [PubMed: 6520251]

Godement P, Salaun J, Mason CA. Retinal axon pathfinding in the optic chiasm: divergence of crossed and uncrossed fibers. Neuron. 1990; 5(2):173-186. [PubMed: 2383400]

Gong S, Doughty M, Harbaugh CR, Cummins A, Hatten ME, Heintz N, Gerfen CR. Targeting Cre recombinase to specific neuron populations with bacterial artificial chromosome constructs. J Neurosci. 2007; 27(37):9817-9823. DOI: 10.1523/JNEUROSCI.2707-07.2007 [PubMed: 17855595]

Graziadei PP, Levine RR, Graziadei GA. Regeneration of olfactory axons and synapse formation in the forebrain after bulbectomy in neonatal mice. Proc Natl Acad Sci U S A. 1978; 75(10):5230-5234. [PubMed: 283428]

Herrera E, Brown L, Aruga J, Rachel RA, Dolen G, Mikoshiba K, Mason CA. Zic2 patterns binocular vision by specifying the uncrossed retinal projection. Cell. 2003; 114(5):545-557. [PubMed: 13678579]

Hornberg H, Cioni JM, Harris WA, Holt CE. Hermes Regulates Axon Sorting in the Optic Tract by Post-Trancriptional Regulation of Neuropilin 1. J Neurosci. 2016; 36(50):12697-12706. DOI: 10.1523/JNEUROSCI.2400-16.2016 [PubMed: 27974617]

Imai T, Sakano H. Axon-axon interactions in neuronal circuit assembly: lessons from olfactory map formation. Eur J Neurosci. 2011; 34(10):1647-1654. DOI: 10.1111/j.1460-9568.2011.07817.x [PubMed: 22103421]

Imai T, Yamazaki T, Kobayakawa R, Kobayakawa K, Abe T, Suzuki M, Sakano H. Pre-target axon sorting establishes the neural map topography. Science. 2009; 325(5940):585-590. DOI: 10.1126/ science.1173596 [PubMed: 19589963]

Kashima DT, Rubel EW, Seidl AH. Pre-target axon sorting in the avian auditory brainstem. J Comp Neurol. 2013; 521(10):2310-2320. DOI: 10.1002/cne.23287 [PubMed: 23239056]

Koch SM, Dela Cruz CG, Hnasko TS, Edwards RH, Huberman AD, Ullian EM. Pathway-specific genetic attenuation of glutamate release alters select features of competition-based visual circuit refinement. Neuron. 2011; 71(2):235-242. DOI: 10.1016/j.neuron.2011.05.045 [PubMed: 21791283] 
Kuwajima T, Sitko AA, Bhansali P, Jurgens C, Guido W, Mason C. ClearT: a detergent- and solventfree clearing method for neuronal and non-neuronal tissue. Development. 2013; 140(6):13641368. DOI: 10.1242/dev.091844 [PubMed: 23444362]

Kuwajima T, Yoshida Y, Takegahara N, Petros T, Kumanogoh A, Jessell TM, Mason CA. Optic Chiasm Presentation of Semaphorin6D in the Context of Plexin-A1 and Nr-CAM Promotes Retinal Axon Midline Crossing. Neuron. 2012; 74(4):676-690. DOI: 10.1016/j.neuron. 2012.03.025 [PubMed: 22632726]

Marcucci F, Murcia-Belmonte V, Wang Q, Coca Y, Ferreiro-Galve S, Kuwajima T, Herrera E. The Ciliary Margin Zone of the Mammalian Retina Generates Retinal Ganglion Cells. Cell Rep. 2016; 17(12):3153-3164. DOI: 10.1016/j.celrep.2016.11.016 [PubMed: 28009286]

Martersteck EM, Hirokawa KE, Evarts M, Bernard A, Duan X, Li Y, Harris JA. Diverse Central Projection Patterns of Retinal Ganglion Cells. Cell Rep. 2017; 18(8):2058-2072. DOI: 10.1016/ j.celrep.2017.01.075 [PubMed: 28228269]

Michalski N, Babai N, Renier N, Perkel DJ, Chedotal A, Schneggenburger R. Robo3-driven axon midline crossing conditions functional maturation of a large commissural synapse. Neuron. 2013; 78(5):855-868. DOI: 10.1016/j.neuron.2013.04.006 [PubMed: 23664551]

Miller AM, Maurer LR, Zou DJ, Firestein S, Greer CA. Axon fasciculation in the developing olfactory nerve. Neural Dev. 2010; 5:20.doi: 10.1186/1749-8104-5-20 [PubMed: 20723208]

Pak W, Hindges R, Lim YS, Pfaff SL, O'Leary DD. Magnitude of binocular vision controlled by islet-2 repression of a genetic program that specifies laterality of retinal axon pathfinding. Cell. 2004; 119(4):567-578. DOI: 10.1016/j.cell.2004.10.026 [PubMed: 15537545]

Petros TJ, Bryson JB, Mason C. Ephrin-B2 elicits differential growth cone collapse and axon retraction in retinal ganglion cells from distinct retinal regions. Dev Neurobiol. 2010; 70(11):781-794. DOI: 10.1002/dneu.20821 [PubMed: 20629048]

Petros TJ, Rebsam A, Mason CA. Retinal axon growth at the optic chiasm: to cross or not to cross. Annu Rev Neurosci. 2008; 31:295-315. DOI: 10.1146/annurev.neuro.31.060407.125609 [PubMed: 18558857]

Plas DT, Lopez JE, Crair MC. Pretarget sorting of retinocollicular axons in the mouse. J Comp Neurol. 2005; 491(4):305-319. DOI: 10.1002/cne.20694 [PubMed: 16175549]

Raper J, Mason C. Cellular Strategies of Axonal Pathfinding. Cold Spring Harb Perspect Biol. 2010; 2(9)doi: 10.1101/cshperspect.a001933

Rebsam A, Petros TJ, Mason CA. Switching retinogeniculate axon laterality leads to normal targeting but abnormal eye-specific segregation that is activity dependent. J Neurosci. 2009; 29(47):1485514863. DOI: 10.1523/JNEUROSCI.3462-09.2009 [PubMed: 19940181]

Reese BE, Baker GE. The re-establishment of the representation of the dorso-ventral retinal axis in the chiasmatic region of the ferret. Vis Neurosci. 1993; 10(5):957-968. [PubMed: 8217945]

Reh TA, Pitts E, Constantine-Paton M. The organization of the fibers in the optic nerve of normal and tectum-less Rana pipiens. J Comp Neurol. 1983; 218(3):282-296. DOI: 10.1002/cne.902180305 [PubMed: 6604077]

Salichon N, Gaspar P, Upton AL, Picaud S, Hanoun N, Hamon M, Seif I. Excessive activation of serotonin (5-HT) 1B receptors disrupts the formation of sensory maps in monoamine oxidase a and 5-ht transporter knock-out mice. J Neurosci. 2001; 21(3):884-896. [PubMed: 11157075]

Seibt J, Schuurmans C, Gradwhol G, Dehay C, Vanderhaeghen P, Guillemot F, Polleux F. Neurogenin2 specifies the connectivity of thalamic neurons by controlling axon responsiveness to intermediate target cues. Neuron. 2003; 39(3):439-452. [PubMed: 12895419]

SitkoAA, , MasonC. Organization of Axons in Their Tracts. In: RocklandK, editorAxons and Brain Architecture1. Academic Press, Elsevier; 2015267288

Smit D, Fouquet C, Pincet F, Zapotocky M, Trembleau A. Axon tension regulates fasciculation/ defasciculation through the control of axon shaft zippering. Elife. 2017; 6doi: 10.7554/eLife.19907

Soares CA, Mason CA. Transient ipsilateral retinal ganglion cell projections to the brain: Extent, targeting, and disappearance. Dev Neurobiol. 2015; doi: 10.1002/dneu.22291

St John JA, Clarris HJ, McKeown S, Royal S, Key B. Sorting and convergence of primary olfactory axons are independent of the olfactory bulb. J Comp Neurol. 2003; 464(2):131-140. DOI: 10.1002/cne.10777 [PubMed: 12898607] 
Torrealba F, Guillery RW, Eysel U, Polley EH, Mason CA. Studies of retinal representations within the cat's optic tract. J Comp Neurol. 1982; 211(4):377-396. DOI: 10.1002/cne.902110405 [PubMed: 7174900]

Upton AL, Salichon N, Lebrand C, Ravary A, Blakely R, Seif I, Gaspar P. Excess of serotonin (5-HT) alters the segregation of ispilateral and contralateral retinal projections in monoamine oxidase $\mathrm{A}$ knock-out mice: possible role of 5-HT uptake in retinal ganglion cells during development. $\mathrm{J}$ Neurosci. 1999; 19(16):7007-7024. [PubMed: 10436056]

Walsh C, Guillery RW. Age-related fiber order in the optic tract of the ferret. J Neurosci. 1985; 5(11): 3061-3069. [PubMed: 3840527]

Wang L, Marquardt T. What axons tell each other: axon-axon signaling in nerve and circuit assembly. Curr Opin Neurobiol. 2013; 23(6):974-982. DOI: 10.1016/j.conb.2013.08.004 [PubMed: 23973157]

Wang LC, Dani J, Godement P, Marcus RC, Mason CA. Crossed and uncrossed retinal axons respond differently to cells of the optic chiasm midline in vitro. Neuron. 1995; 15(6):1349-1364. DOI: 10.1016/0896-6273(95)90013-6 [PubMed: 8845158]

Wang LC, Rachel RA, Marcus RC, Mason CA. Chemosuppression of Retinal Axon Growth by the Mouse Optic Chiasm. Neuron. 1996; 17(5):849-862. DOI: 10.1016/S0896-6273(00)80217-2 [PubMed: 8938118]

Wang Q, Marcucci F, Cerullo I, Mason C. Ipsilateral and Contralateral Retinal Ganglion Cells Express Distinct Genes during Decussation at the Optic Chiasm. eNeuro. 2016; 3(6)doi: 10.1523/ ENEURO.0169-16.2016

Weth F, Fiederling F, Gebhardt C, Bastmeyer M. Chemoaffinity in topographic mapping revisited - Is it more about fiber-fiber than fiber-target interactions? Semin Cell Dev Biol. 2014; doi: 10.1016/ j.semcdb.2014.07.010

Williams SE, Grumet M, Colman DR, Henkemeyer M, Mason CA, Sakurai T. A role for Nr-CAM in the patterning of binocular visual pathways. Neuron. 2006; 50(4):535-547. DOI: 10.1016/j.neuron. 2006.03.037 [PubMed: 16701205]

Williams SE, Mann F, Erskine L, Sakurai T, Wei S, Rossi DJ, Henkemeyer M. Ephrin-B2 and EphB1 mediate retinal axon divergence at the optic chiasm. Neuron. 2003; 39(6):919-935. [PubMed: 12971893]

Zhou J, Wen Y, She L, Sui YN, Liu L, Richards LJ, Poo MM. Axon position within the corpus callosum determines contralateral cortical projection. Proc Natl Acad Sci U S A. 2013; 110(29):E2714-2723. DOI: 10.1073/pnas.1310233110 [PubMed: 23812756] 

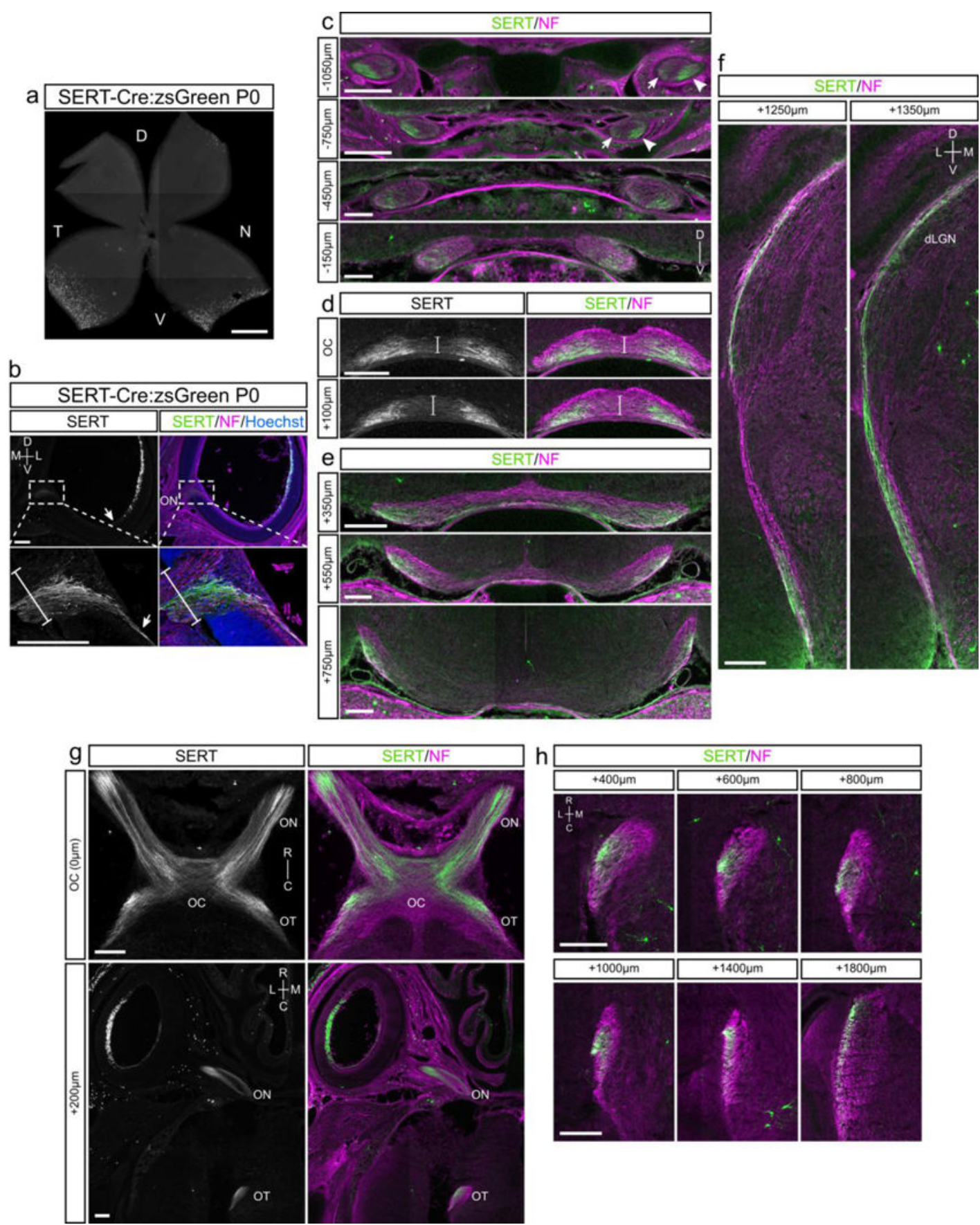

Figure 1. Genetically labeled ipsilateral RGC axons are segregated to varying degrees in the optic nerve, chiasm, and tract

Retina (a) and $25 \mu \mathrm{m}$ thick cryosections (b-h) from P0 SERT-Cre::zsGreen mice, immunostained for zsGreen (green), labeling SERT+ cells and axons, and neurofilament (NF, magenta), labeling all axons. (a) In a flat-mounted retina, SERT-expressing cells are evident in the ventrotemporal (VT) retina, the source of ipsilateral RGCs. (b-h) Cryosections in b-f are in the frontal plane, and in $\mathbf{g - h}$, the horizontal plane. In b-f, the optic chiasm (OC) is designated as $0 \mu \mathrm{m}$, sections rostral to the $\mathrm{OC}$ are indicated by $-\mu \mathrm{m}$ from the $\mathrm{OC}$, and 
sections caudal to the $\mathrm{OC}$ are indicated by $+\mu \mathrm{m}$. In $\mathbf{g - h}$, the $\mathrm{OC}$ is $0 \mu \mathrm{m}$ and sections dorsal to the OC are $+\mu \mathrm{m}$. (b) In the VT retina, zsGreen+ cells are in the RGC layer (top panel) and send their axons (arrows) into the optic nerve (ON). Insets show zsGreen+ axons exiting the retina and entering the optic stalk in a ventral position. Bracket shows width of the proximal ON based on NF labeling. (c) ZsGreen+ RGC axons are mainly in a ventrolateral position (arrowheads), with a subpopulation in the medial ON (arrows). As the two ONs near the midline to merge at the OC, zsGreen+ axons become less clearly bundled together (bottom panels). (d) ZsGreen+ axons interact with nearly the full dorsoventral extent of the $\mathrm{OC}$ as they turn away to project ipsilaterally. Brackets indicate span of zsGreen+ axons at the midline. (e) Caudal to the OC, axons enter the optic tract (OT) and zsGreen+ axons rebundle in the lateral OT. (f) ZsGreen+ axons maintain a lateral position along the full extent of the OT as they reach the dorsal lateral geniculate nucleus (dLGN). (g) Top, horizontal section showing the ON, OC, and OT. Bottom, horizontal section showing the retina, ON, and OT. (h) Sequential horizontal sections through the OT demonstrate the localization of zsGreen+ axons to the lateral aspect of the tract. Scale bars: $500 \mu \mathrm{m}$ (a), $200 \mu \mathrm{m}(\mathbf{b}-\mathbf{h})$. D = dorsal, $\mathrm{V}=$ ventral, $\mathrm{T}=$ temporal, $\mathrm{N}=$ nasal, $\mathrm{M}=$ medial, $\mathrm{L}=$ lateral, $\mathrm{ON}=$ optic nerve, $\mathrm{OC}$ $=$ optic chiasm, $\mathrm{OT}=$ optic tract, $\mathrm{dLGN}=$ dorsal lateral geniculate nucleus, $\mathrm{NF}=$ neurofilament. 
a

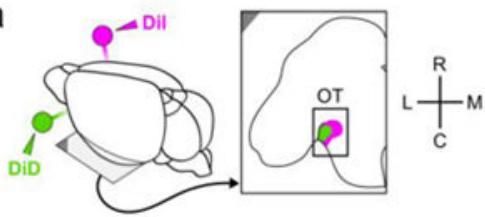

b
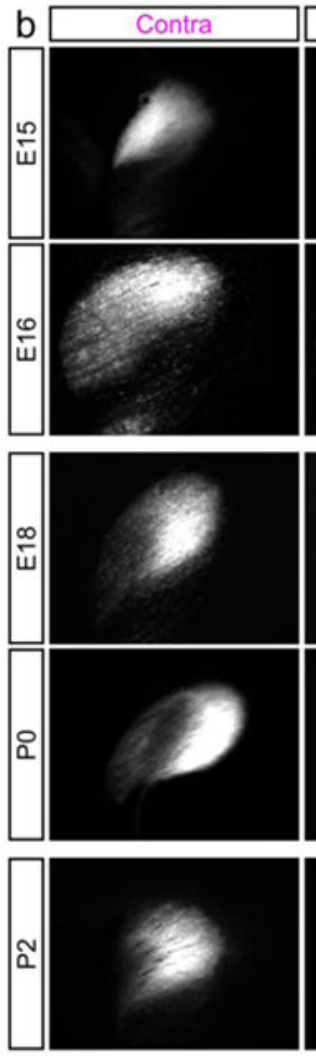
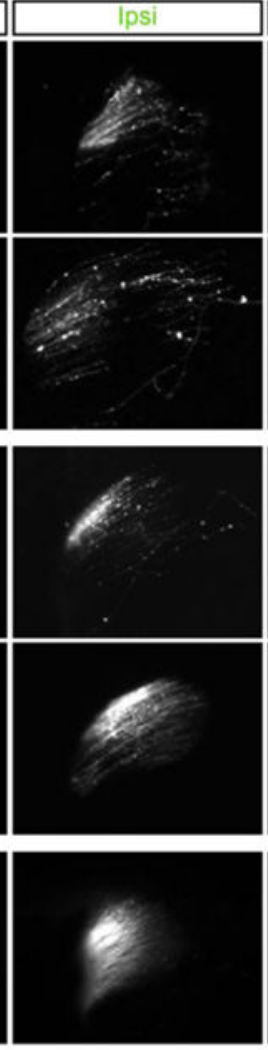
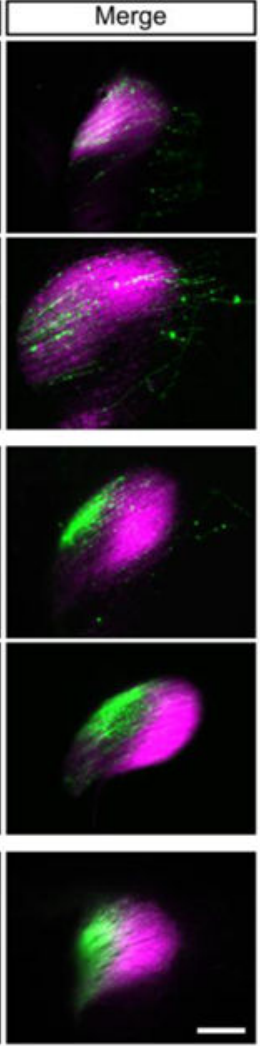

d

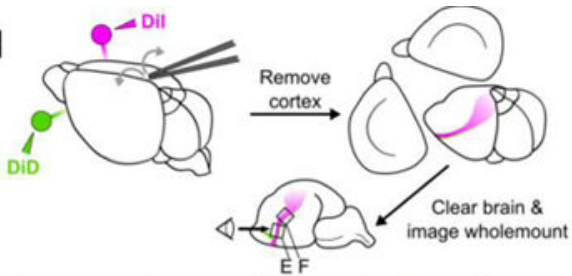

e
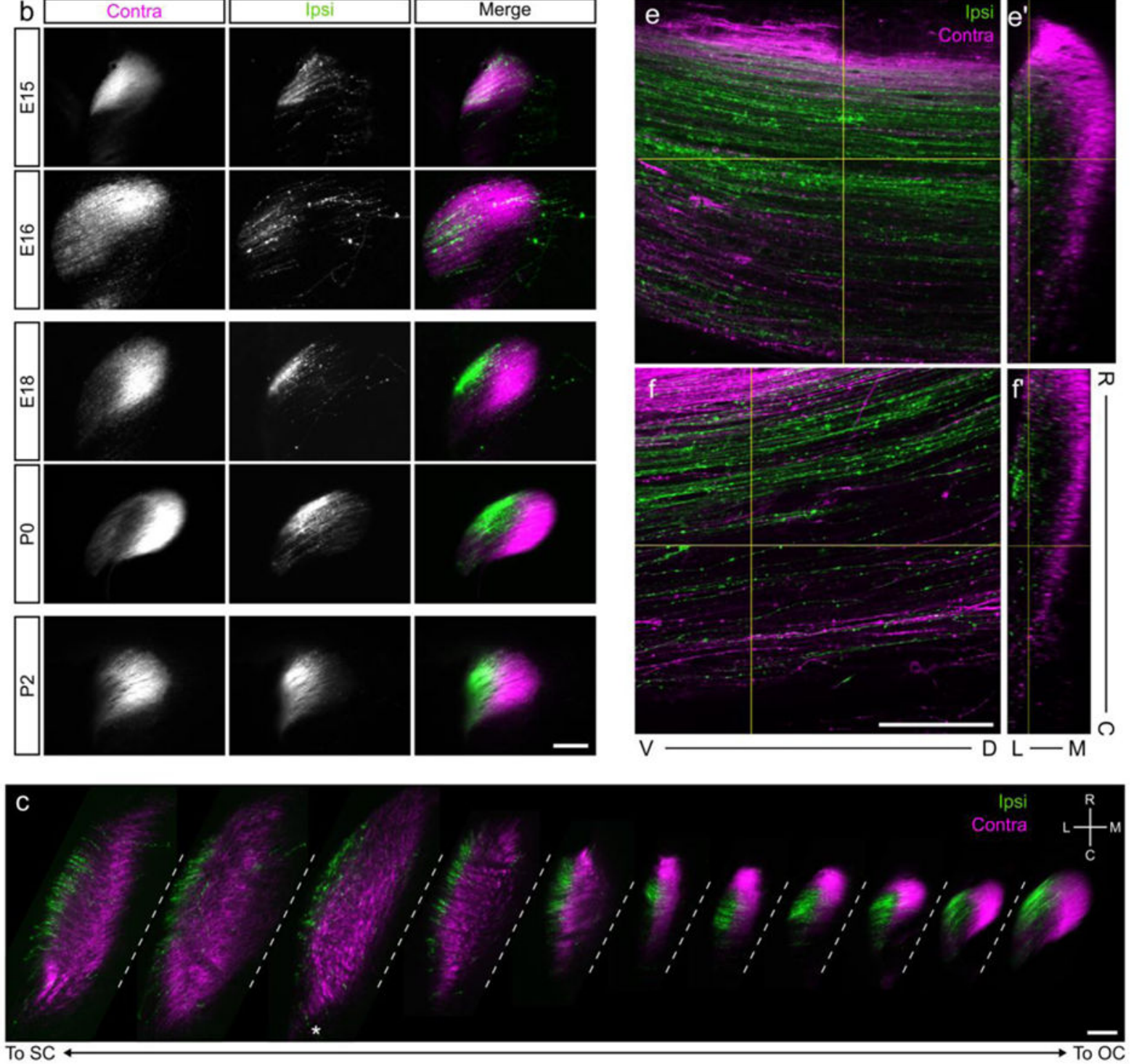

Figure 2. Ipsilateral and contralateral RGC axons are segregated in the optic tract (a) Anterograde labeling approach: DiI (pseudocolored magenta) and DiD (pseudocolored green) crystals were inserted onto the optic nerve heads of the right and left eyes, respectively, of fixed wild-type embryonic or postnatal heads. Brains with labeled optic tracts (OTs) were sectioned $75 \mu \mathrm{m}$ thick in the horizontal plane. (b) Developmental series of anterograde labeling of ipsilateral and contralateral RGC axons in the OT. Sections are approximately $150 \mu \mathrm{m}$ dorsal to the optic chiasm. Ipsilateral axons are situated in the lateral OT throughout development, largely segregated from contralateral axons. (c) Serial sections 
through the OT at P0. Approximately every third section is shown in sequence, starting ventrally at right, approximately $75-150 \mu \mathrm{m}$ dorsal to the optic chiasm, and extending dorsally leftwards towards the SC. Asterisk indicates the beginning of the dLGN. Ipsilateral RGC axons persist in the lateral OT throughout the length of the OT. (d) Wholemount imaging approach: after DiI/DiD labeling, the cortex was removed and brain was cleared using Clear $^{T}$ (Kuwajima et al., 2013). (e) One optical slice of the proximal OT, and corresponding orthogonal view of z-stack (e'). (f) One optical slice of the distal OT, and corresponding orthogonal view of z-stack (f'). These high-resolution views of the OT show that while some individual ipsi- and contralateral fibers intermingle, the two populations are grossly segregated. Movie 1 corresponds to e, Movie 2 corresponds to $\mathbf{f}$. Scale bars: $100 \mu \mathrm{m}$. $\mathrm{D}=$ dorsal, $\mathrm{V}=$ ventral, $\mathrm{M}=$ medial, $\mathrm{L}=$ lateral, $\mathrm{R}=$ rostral, $\mathrm{C}=$ caudal, $\mathrm{OT}=$ optic tract, $\mathrm{OC}$ $=$ optic chiasm, $\mathrm{SC}=$ superior colliculus. 
a
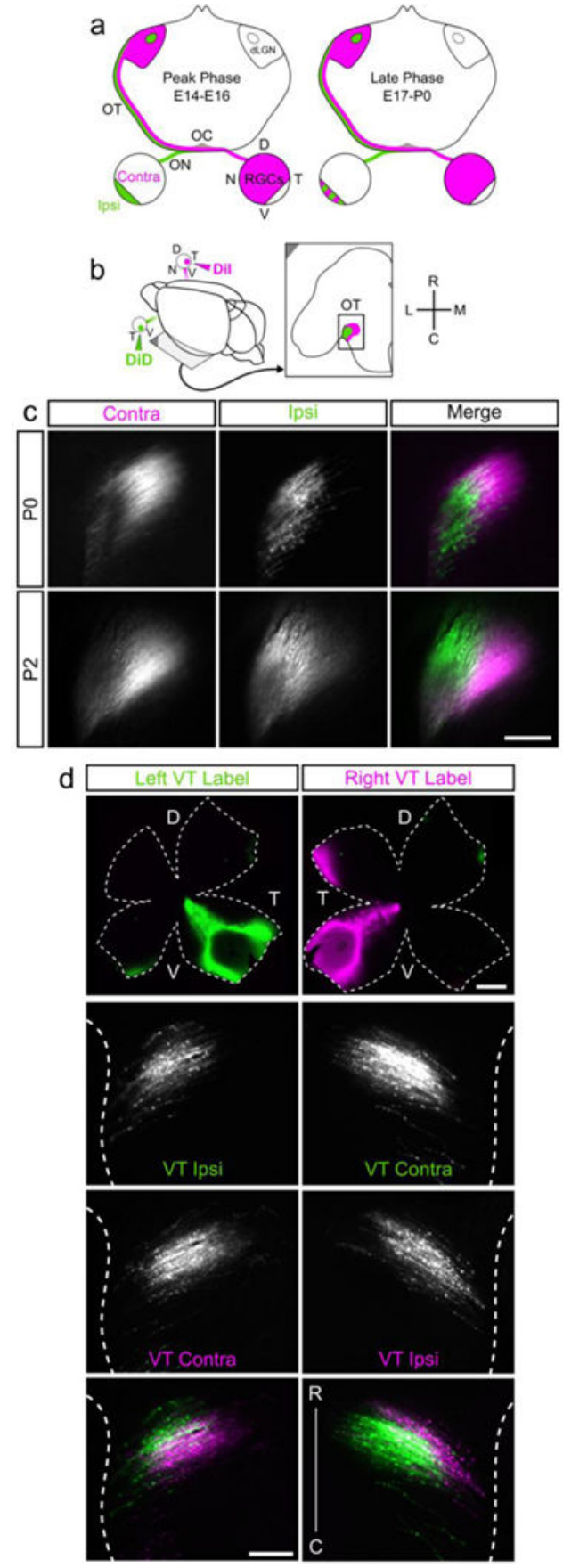

Figure 3. Ipsilateral and contralateral RGC axons arising from the same topographic region segregate in the optic tract

(a) Developmental timing of ipsilateral and contralateral RGC axon outgrowth. During the peak phase of RGC axon outgrowth (left), VT retina produces ipsilateral RGCs. Later, from E17 to birth (right), late-born contralateral RGCs are born in VT retina, in addition to ipsilateral RGCs. (b) Focal anterograde labeling approach: DiI and DiD were placed in the VT region of each retina of fixed wild-type pups, and then sectioned $75 \mu \mathrm{m}$ thick in the horizontal plane to examine the OT. Box around OT indicates area shown in micrographs of 
the OT in c-d. (c) Horizontal sections approximately $150 \mu \mathrm{m}$ dorsal to the OC at P0 and P2 with focal VT/VT DiI/DiD labeling. Ipsilateral axons from VT retina are positioned laterally to contralateral axons arising from the same topographic region. (d) Retinae (top) and both hemispheres of the OT (bottom panels) from a P0 mouse with VT/VT label as indicated in (b). Both ipsilateral and contralateral labeling is shown for both retinae, for direct comparison. Dashed lines indicate the lateral edge of the thalamus. Ipsilateral axons are positioned laterally and slightly rostrally to contralateral axons arising from VT retina. Scale bars: $500 \mu \mathrm{m}$ (d, top), $100 \mu \mathrm{m}$ (c; d, bottom). $\mathrm{D}=$ dorsal, $\mathrm{V}=$ ventral, $\mathrm{N}=$ nasal, $\mathrm{T}=$ temporal, $\mathrm{M}=$ medial, $\mathrm{L}=$ lateral, $\mathrm{R}=$ rostral, $\mathrm{C}=$ caudal, $\mathrm{RGCs}=$ retinal ganglion cells, $\mathrm{ON}$ $=$ optic nerve, $\mathrm{OC}=$ optic chiasm, $\mathrm{OT}=$ optic tract, $\mathrm{dLGN}=$ dorsal lateral geniculate nucleus. 

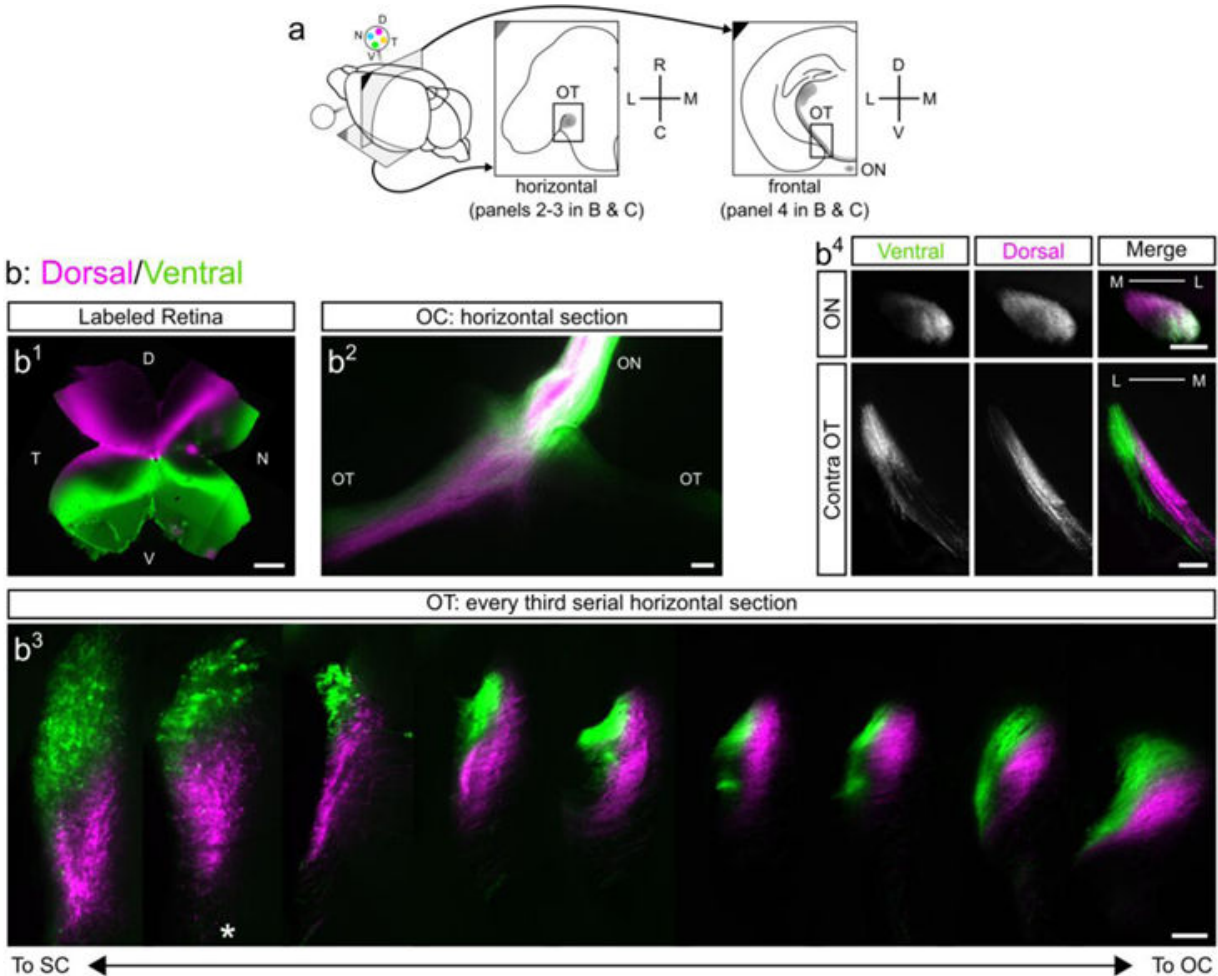

c: Tempora/Nasal
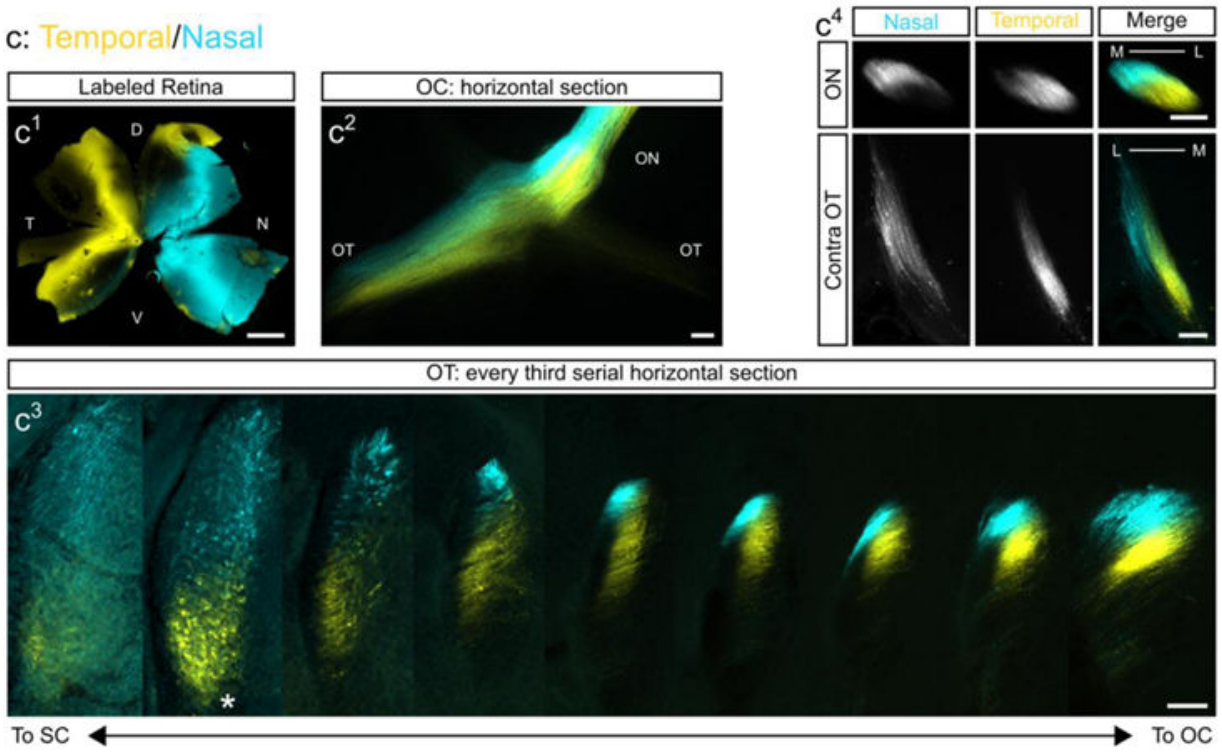

Figure 4. RGC axons are topographically organized in the retinogeniculate pathway

(a) Schematic of labeling and sectioning approach. DiI and DiD were used to label dorsal and ventral (magenta and green, respectively) hemiretinae of fixed P0 wild-type pups, shown in (b), or nasal and temporal (cyan and yellow, respectively) hemiretinae, shown in (c). Labeled brains were sectioned $75 \mu \mathrm{m}$ thick in either the horizontal or frontal plane. ( $\mathbf{b}^{\mathbf{1}}$ ) and $\left(\mathbf{c}^{\mathbf{1}}\right)$ show representative labeled retinae. $\left(\mathbf{b}^{\mathbf{2}}\right)$ and $\left(\mathbf{c}^{\mathbf{2}}\right)$ show a horizontal section through the optic chiasm, with the optic nerve at top and the optic tracts at bottom. $\left(\mathbf{b}^{\mathbf{3}}\right)$ and $\left(\mathbf{c}^{\mathbf{3}}\right)$ show serial horizontal sections through the OT at P0. Every third section is shown in sequence, 
starting ventrally at right, approximately $75-150 \mu \mathrm{m}$ dorsal to the optic chiasm, and extending dorsally leftwards towards the SC. Asterisk indicates the beginning of the dLGN. $\left(b^{\mathbf{1 - 3}}\right)$ are from the same animal; $\left(\mathbf{c}^{\mathbf{1 - 3}}\right)$ are from the same animal. $\left(\mathbf{b}^{\mathbf{4}}\right)$ and $\left(\mathbf{c}^{\mathbf{4}}\right)$ show frontal sections through the optic nerve (top) and optic tract (bottom). The optic nerves are ipsilateral to the labeled retina, the optic tracts are contralateral to the labeled retina. Scale bars: $500 \mu \mathrm{m}\left(\mathbf{b}^{\mathbf{1}}, \mathbf{c}^{\mathbf{1}}\right) 100 \mu \mathrm{m}\left(\mathbf{b}^{\mathbf{2 - 4}}, \mathbf{c}^{\mathbf{2 - 4}}\right) . \mathrm{D}=$ dorsal, $\mathrm{V}=$ ventral, $\mathrm{N}=$ nasal, $\mathrm{T}=$ temporal, $\mathrm{M}=$ medial, $\mathrm{L}=$ lateral, $\mathrm{R}=$ rostral, $\mathrm{C}=$ caudal, $\mathrm{RGCs}=$ retinal ganglion cells, $\mathrm{ON}=$ optic nerve, $\mathrm{OC}=$ optic chiasm, $\mathrm{OT}=$ optic tract, $\mathrm{SC}=$ superior colliculus . 

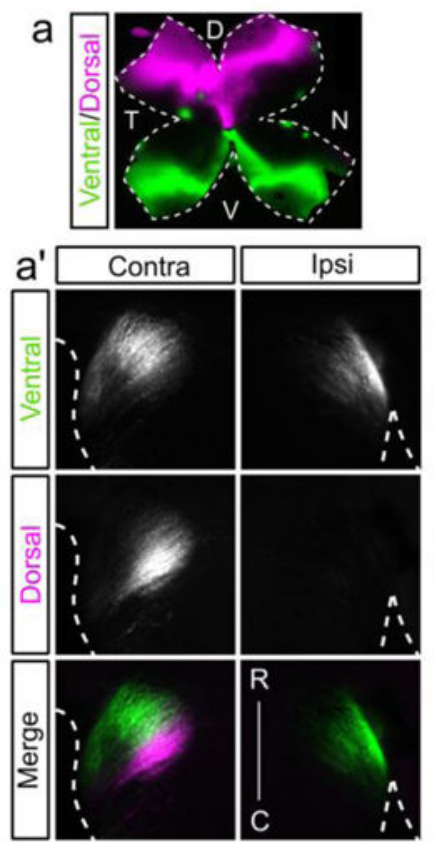
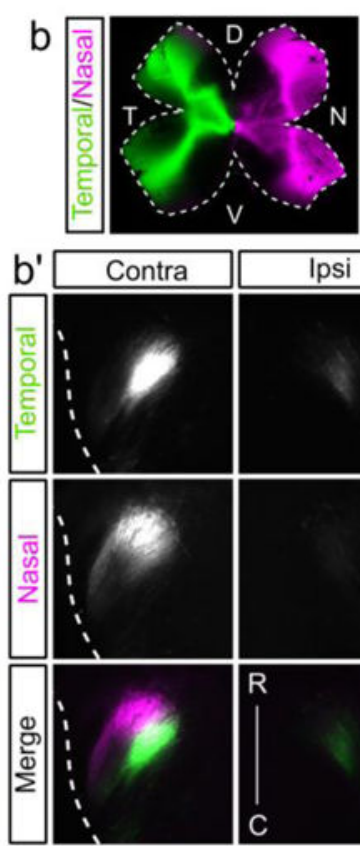

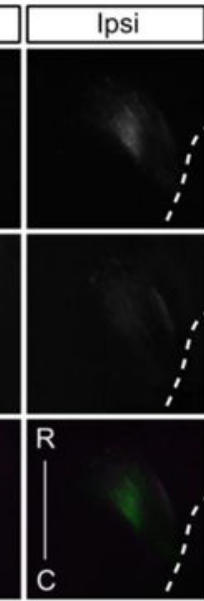

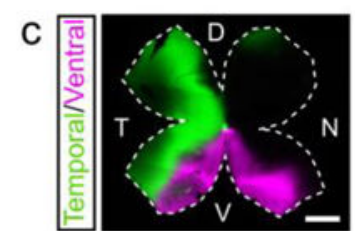
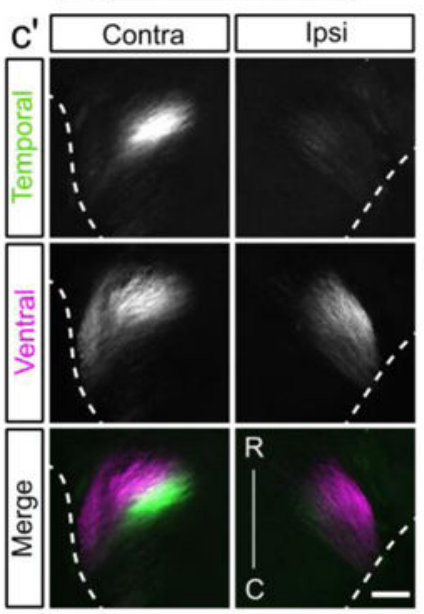

d
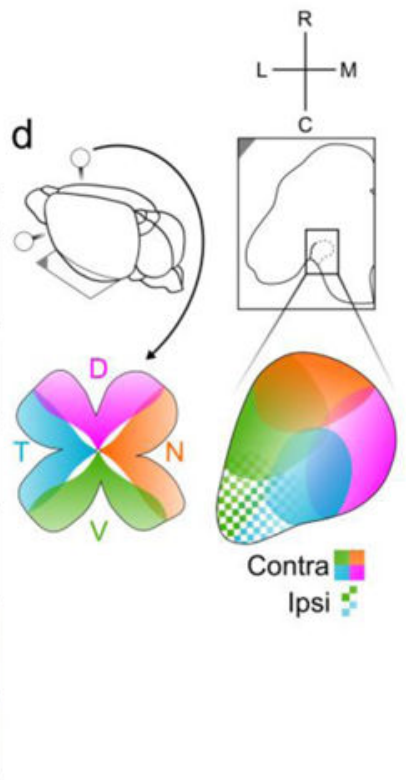

Figure 5. Ipsilateral axons are offset laterally from the contralateral topographic map Focal DiI/DiD labeling in the right retinae of fixed P0 wild-type pups $(\mathbf{a}, \mathbf{b}, \mathbf{c})$, and corresponding $75 \mu \mathrm{m}$ thick horizontal sections of both ipsilateral and contralateral optic tracts, approximately $150 \mu \mathrm{m}$ dorsal to the optic chiasm (a', b', c'). Dashed lines indicate the lateral edge of the thalamus. (a, a') Dorsal RGC axons (magenta) are in the caudomedial aspect of the optic tract. Ventral RGC axons (green) are in the rostrolateral aspect of the contralateral tract, and positioned more laterally in the ipsilateral tract. (b, b') Nasal (magenta) and temporal (green) RGC axons are segregated across the rostrocaudal aspect of the optic tract, respectively. Temporal RGC axons in the ipsilateral tract are positioned more laterally and slightly more caudally than in the contralateral tract. (c, $\left.\mathbf{c}^{\prime}\right)$ Neighboring ventral (magenta) and temporal (green) RGC axons are segregated along a rostrolateral to caudomedial axis in the optic tract, respectively. The two populations in the ipsilateral tract are similarly segregated, with a slightly more lateral position relative to the contralateral tract. (d) Summary of topographic position of RGC axons in the optic tract. Bottom left shows the color codes of RGCs labeled in a flat-mounted retina and bottom right shows the relative distribution of RGC axons in a horizontal section through the optic tract approximately $150 \mu \mathrm{m}$ dorsal to the optic chiasm. The contralateral topographic map is shown in solid color gradients, and ipsilateral axon positions are overlaid with a checkered pattern. Scale bars: $500 \mu \mathrm{m}(\mathbf{a}, \mathbf{b}, \mathbf{c})$ and $100 \mu \mathrm{m}\left(\mathbf{a}^{\prime}, \mathbf{b}^{\prime}, \mathbf{c}^{\prime}\right) . \mathrm{D}=$ dorsal, $\mathrm{V}=$ ventral, $\mathrm{N}=$ nasal, $\mathrm{T}=$ temporal, $\mathrm{M}=$ medial, $\mathrm{L}=$ lateral, $\mathrm{R}=$ rostral, $\mathrm{C}=$ caudal. 


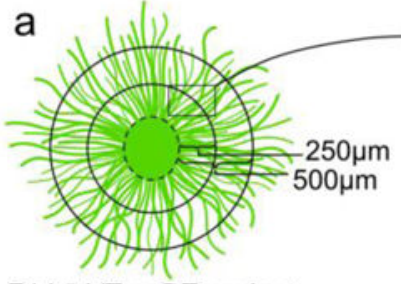

E14.5 VT or DT explant

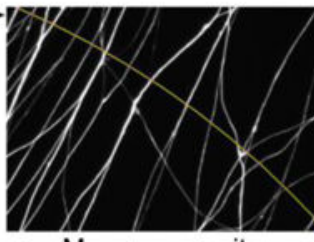

Measure neurite bundle widths

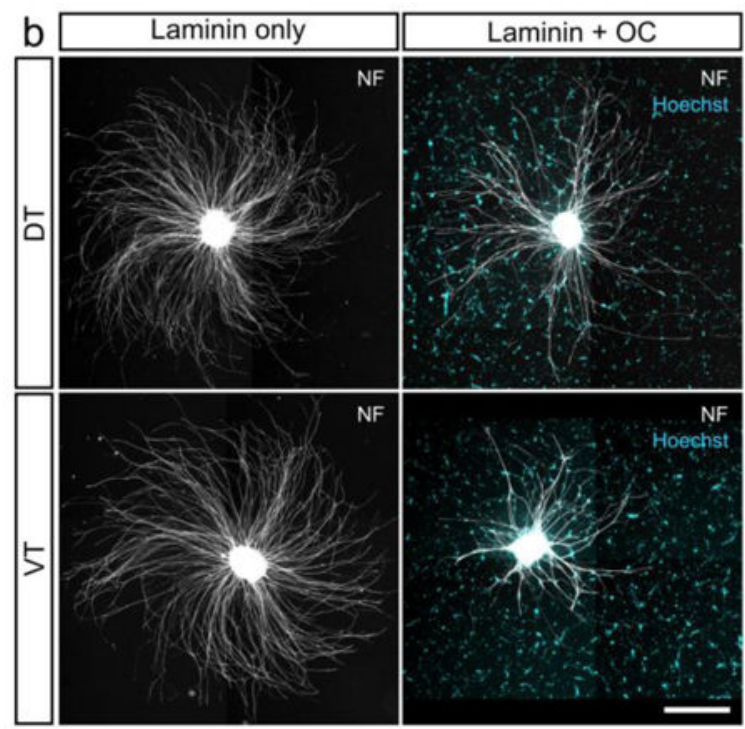

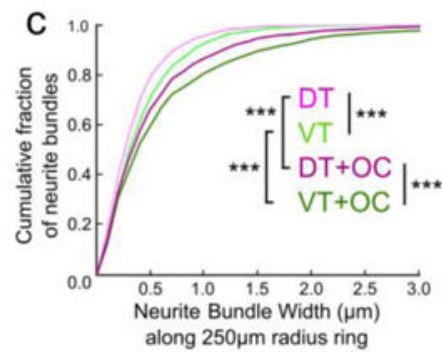
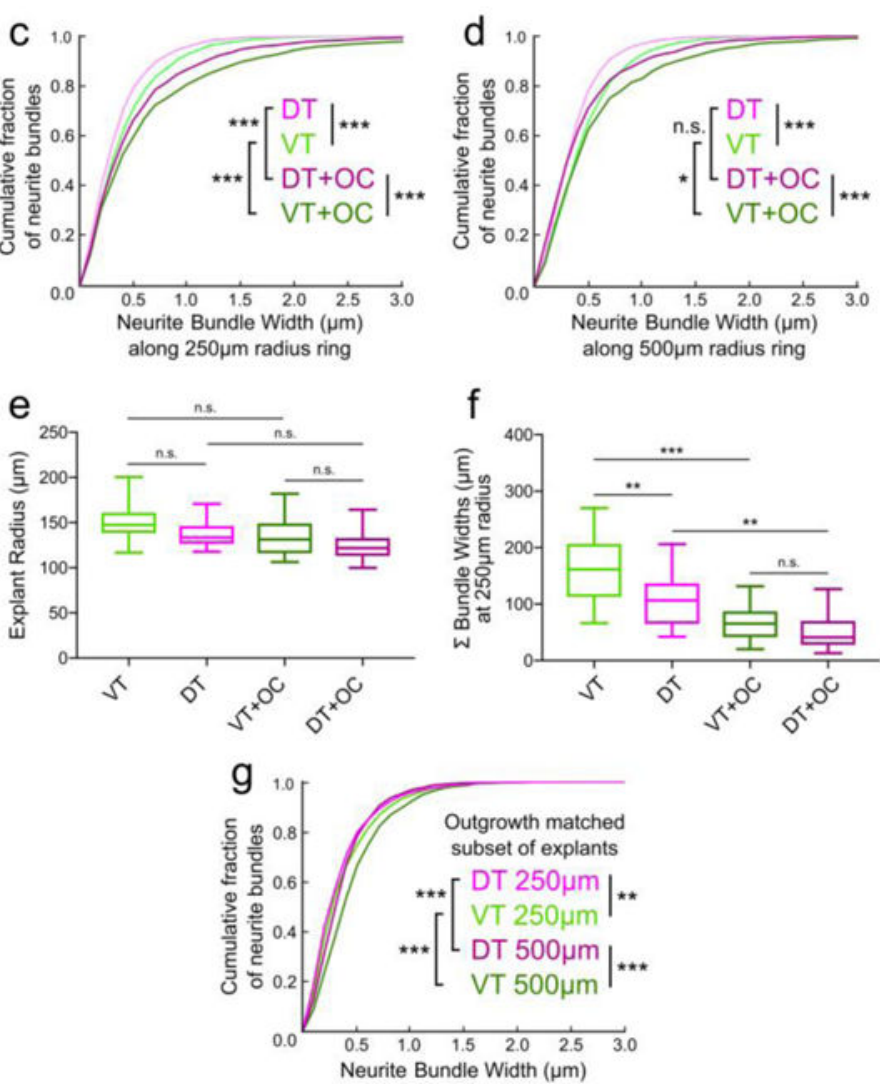

Figure 6. Neurites from VT retina have an intrinsic preference to grow and fasciculate more than neurites from DT retina

(a) Retinal explant analysis of neurite bundle width. Explants were taken from E14.5 wildtype retinae, from either the VT (ipsilateral RGCs) or DT (contralateral RGCs) retina and cultured for $24 \mathrm{~h}$, then fixed and analyzed. Two rings were drawn $250 \mu \mathrm{m}$ and $500 \mu \mathrm{m}$ away from the perimeter of the explant body. The width of each neurite bundle was measured as it intersected the ring. (b) Representative explants from DT and VT retina, grown on laminin only (left column) or laminin and dissociated chiasm cells (OC, right column, labeled with Hoechst in blue). Explants are immunostained for neurofilament (NF, white). (c-d) Cumulative frequency distributions (CFD) of neurite bundle widths around the $250 \mu \mathrm{m}$ ring (c) and the $500 \mu \mathrm{m}$ ring (d), in laminin only (light green and light magenta) and laminin + chiasm cells (dark green and dark magenta) growth conditions. (c) Around the $250 \mu \mathrm{m}$ ring, VT neurites form thicker bundles than DT neurites when grown on laminin alone (light green and light magenta lines, respectively, $* * * \mathrm{p}=3.85 \times 10^{-22}$, Mann Whitney $U$ test), and when grown on laminin with dissociated chiasm cells (dark green and dark magenta lines, respectively, $* * * \mathrm{p}=3.23 \times 10^{-4}$, Mann Whitney $U$ test). DT neurites grown on chiasm cells form thicker bundles than those grown on laminin only (dark and light magenta lines, respectively, $* * * \mathrm{p}=6.76 \times 10^{-21}$, Mann Whitney $U$ test). VT neurites grown on chiasm cells form thicker bundles than those grown on laminin only (dark and light green lines, respectively, $* * * \mathrm{p}=7.24 \times 10^{-18}$, Mann Whitney $U$ test). (d) Around the $500 \mu \mathrm{m}$ ring, the differences between VT and DT neurites are maintained in both laminin only $(* * * \mathrm{p}=1.29 \times$ $10^{-42}$, Mann Whitney $U$ test) and laminin + chiasm cell $\left(* * * \mathrm{p}=4.46 \times 10^{-8}\right.$, Mann Whitney 
$U$ test) growth conditions. VT neurites grown with chiasm cells formed thicker bundles at the $500 \mu \mathrm{m}$ ring than those grown only on laminin ( ${ }^{*} \mathrm{p}=0.029$, Mann Whitney $U$ test).

However, the difference between bundle widths of DT neurites cultured alone versus chiasm co-culture failed to reach statistical significance ( $\mathrm{p}=0.11$, Mann Whitney $U$ test). $\mathrm{n}=17$ explants from 4 experiments (DT), 18 explants from 4 experiments (VT), 16 explants from 3 experiments (DT+OC and VT+OC). (e) Explant size, indicated by radius of the explant body, was consistent across explant type and growth condition $(\mathrm{p}=0.15$, VT vs DT; $\mathrm{p}=$ $0.50, \mathrm{VT}+\mathrm{OC}$ vs DT+OC; $\mathrm{p}=0.09$, VT vs VT+OC; $\mathrm{p}=0.29$, DT vs DT+OC; One-Way ANOVA, Holm-Sidak's multiple comparison test). (f) Neurite outgrowth, measured as the sum of all bundle widths along the $250 \mu \mathrm{m}$ ring, varied across explant type and growth condition. VT explants had greater neurite outgrowth than DT explants when grown on laminin (**p $=0.0073$, One-Way ANOVA), but not in the chiasm co-culture condition ( $\mathrm{p}=$ 0.4 , One-Way ANOVA). Both explant types had stunted outgrowth in the chiasm co-culture condition $(* * * \mathrm{p}<0.0001$, VT vs VT+OC; ** $\mathrm{p}=0.002$, DT vs DT+OC, One-Way ANOVA, Holm-Sidak's multiple comparison test). (g) CFD of a subset of VT and DT explants grown on laminin alone, which are matched for total neurite outgrowth. VT $(n=5$ explants from 3 experiments) and DT ( $\mathrm{n}=6$ explants from 3 experiments) explants that had total neurite outgrowth (measured along the $250 \mu \mathrm{m}$ ring) between 90-150 $\mu \mathrm{m}$ were chosen from the full data set. Within this subset of explants, VT neurites still formed thicker bundles around both the $250 \mu \mathrm{m}$ and $500 \mu \mathrm{m}$ rings (light and dark green, respectively) than DT neurites, when grown on laminin alone $\left(* * \mathrm{p}=0.0017,250 \mu \mathrm{m}\right.$ ring; $* * * \mathrm{p}=1.64 \times 10^{-14}, 500 \mu \mathrm{m}$ ring; Mann Whitney $U$ test). Both explant types form thicker bundles at the $500 \mu \mathrm{m}$ ring compared to the $250 \mu \mathrm{m}$ ring $\left(* * * \mathrm{p}=4.55 \times 10^{-16}\right.$, VT neurites; $* * * \mathrm{p}=1.86 \times 10^{-7}$, DT neurites; Mann Whitney $U$ test). Box and whisker plots show medians with whiskers extending to the minimum and maximum. CFD plots terminate at $3 \mu \mathrm{m}$ along the $\mathrm{x}$-axis for clarity, although data extend to $4+\mu \mathrm{m}$. Scale bar: $500 \mu \mathrm{m}$. VT $=$ ventrotemporal retina, $\mathrm{DT}=$ dorsotemporal retina, $\mathrm{OC}=$ optic chiasm cells, $\mathrm{NF}=$ neurofilament. 

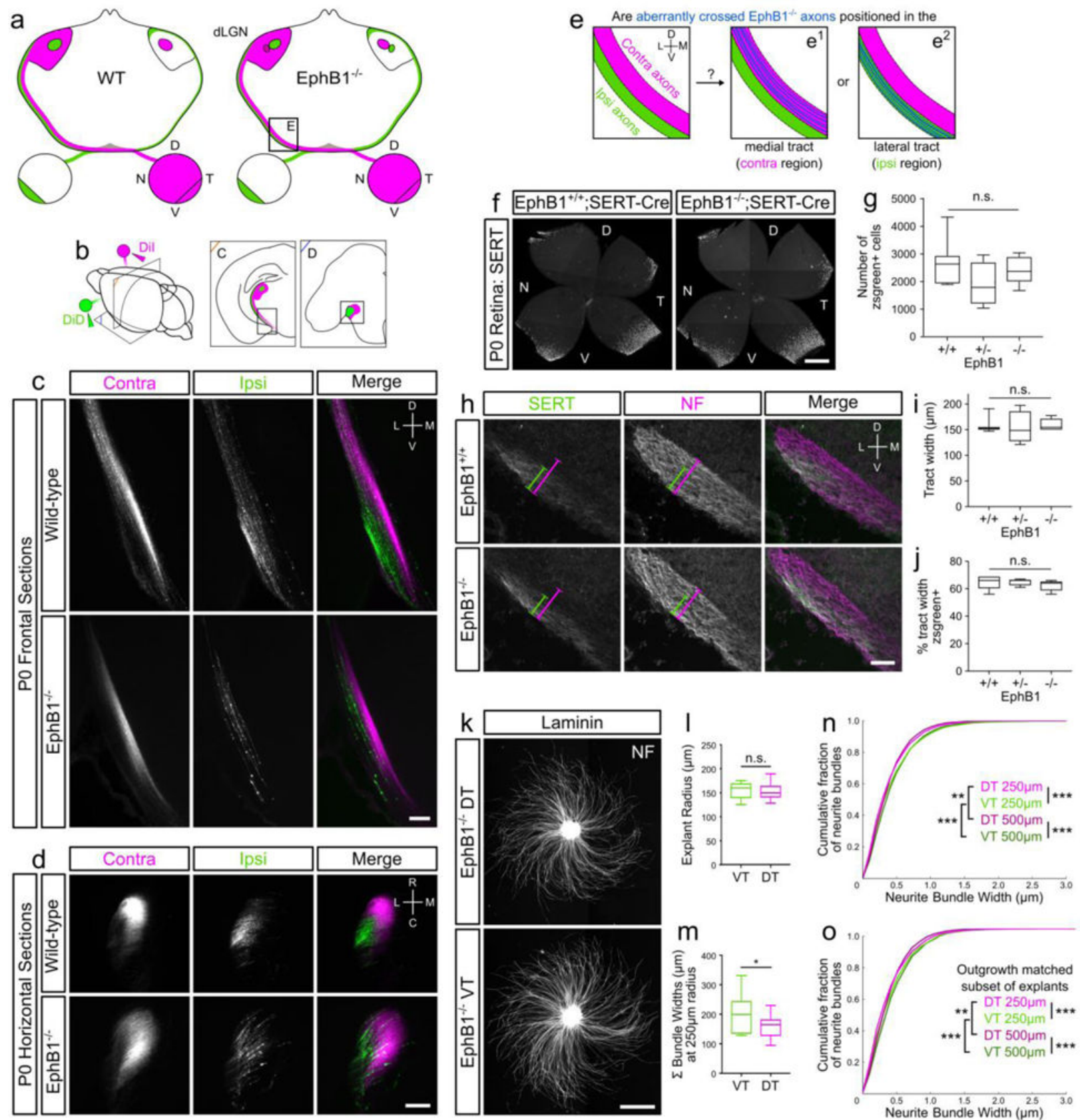

Figure 7. Ipsilateral self-association is preserved in vivo and in vitro in the absence of EphB1 (a) Schematic of eye-specific RGC axon targeting in the dLGN of wild-type and EphB1 $1^{-/-}$ mutant mice. Late-born contralateral RGCs in VT retina (see Fig. 3a) target the dorsal tip of the contralateral $\mathrm{dLGN}$. In the EphB1 ${ }^{-/-}$mutant, many ipsilateral RGC axons are misrouted at the optic chiasm and aberrantly project contralaterally. However, in the dLGN, the misrouted axons target the ipsilateral zone, rather than the dorsal tip. Box corresponds to panel (e). (b) Schematic of labeling and sectioning approach, showing both frontal and horizontal sections. Boxes in cartoon sections indicate area of view in (c) and (d). (c-d) Frontal (c) and horizontal (d) sections through the optic tract of P0 wild-type (top rows) and 
$\mathrm{EphB1}^{-/-}$(bottom rows) pups. Remaining ipsilateral RGC axons in the EphB1 ${ }^{-/-}$tract retain a lateral position, but appear poorly fasciculated and slightly disorganized. (e) Schematic of the optic tract showing the ipsilateral zone (green) in the lateral tract, and the contralateral zone (magenta) in the medial tract. Aberrantly crossed axons in the EphB1 $1^{-/-}$could be positioned with contralateral axons $\left(\mathbf{e}^{\mathbf{1}}\right)$ or retain an association with ipsilateral axons $\left(\mathbf{e}^{\mathbf{2}}\right)$. (f) Representative flat-mounted retinae from SERT;EphB1 ${ }^{+/+}$(left) and SERT;EphB1 ${ }^{-/-}$ (right) P0 pups. (g) There is no difference in zsGreen+ cell counts performed in flatmounted retinae between EphB1 genotypes (mean \pm SEM: SERT;EphB $1^{+/+}=2703 \pm 315.4$; SERT;EphB1 ${ }^{+/-}=1896 \pm 403 ;$ SERT;EphB1 ${ }^{-/-}=2404 \pm 203.7 ; \mathrm{p}=0.24$, One-Way ANOVA; $\mathrm{n}=7$ SERT;EphB1 ${ }^{+/+}$mice, 4 SERT;EphB1 ${ }^{+/-}$mice, 6 SERT;EphB1 ${ }^{-/-}$mice, from 3 litters). (h) Representative $25 \mu \mathrm{m}$ thick frontal sections through the optic tract of SERT;EphB1 ${ }^{+/+}$(top) and SERT;EphB1 ${ }^{-/-}$(bottom) P0 pups. Green brackets indicate span of zsGreen+ axons in the tract; magenta brackets indicate full width of the optic tract, based on neurofilament (NF) staining. There is no difference in total tract width (i) or the percent of tract occupied by zsGreen+ axons (j) between EphB1 genotypes. Mean tract widths \pm SEM: SERT;EphB1 ${ }^{+/+}=157.5 \mu \mathrm{m} \pm 6.1$; SERT;EphB1 $1^{+/-}=154.0 \mu \mathrm{m} \pm 16.0$; SERT;EphB1 ${ }^{-/-}=159.6 \mu \mathrm{m} \pm 5.5 ; \mathrm{p}=0.91$, One-Way ANOVA. Mean \pm SEM percent of tract occupied by zsGreen+ fibers: SERT;EphB $1^{+/+}=64 \% \pm 2.0$; SERT;EphB1 $1^{+/-}=65 \%$ \pm 1.4 ; SERT;EphB1 $1^{-/-}=62.4 \% \pm 1.8 ; \mathrm{p}=0.67$, One-Way ANOVA. $\mathrm{n}=7$ SERT;EphB $1^{+/+}$ mice, 4 SERT;EphB1 $1^{+/-}$mice, 5 SERT;EphB1 ${ }^{-/-}$mice, from 4 litters. (k) Representative DT and VT explants from E14.5 EphB1 ${ }^{-/-}$retinae grown for $24 \mathrm{~h}$ on laminin and immunostained for NF. Explants were analyzed in the same fashion as before (see Fig. 6A). (l) There was no difference in explant size between EphB1 ${ }^{-/-}$VT and DT explants, measured as radius of the explant body (mean \pm SEM: VT $=153.4 \mu \mathrm{m} \pm 4.6$; $\mathrm{DT}=152.8 \mu \mathrm{m} \pm 3.8 ; \mathrm{p}=0.99$, OneWay ANOVA). (m) EphB1 $1^{-/-}$VT explants extended more neurites than EphB1 ${ }^{-/-}$DT explants, measured as the sum of bundle widths around the $250 \mu \mathrm{m}$ ring (mean \pm SEM: VT $=201.4 \mu \mathrm{m} \pm 15.1 ; \mathrm{DT}=160.5 \mu \mathrm{m} \pm 8.4 ; * \mathrm{p}=0.037$, One-Way ANOVA). (n) Cumulative frequency distribution (CFD) of EphB1 $1^{-/} \mathrm{VT}$ and $\mathrm{EphB} 1^{-/-} \mathrm{DT}$ neurite bundle widths intersecting rings placed $250 \mu \mathrm{m}$ (light green and light magenta, respectively) and $500 \mu \mathrm{m}$ (dark green and dark magenta, respectively) away from the perimeter of the explant body. At both distances from the explant body, EphB1 ${ }^{-1-}$ VT neurites form thicker bundles than EphB1 ${ }^{-/-}$DT neurites $\left(* * * \mathrm{p}=1.20 \times 10^{-7}, 250 \mu \mathrm{m}\right.$ ring; $* * * \mathrm{p}=2.74 \times 10^{-14}, 500 \mu \mathrm{m}$ ring; Mann Whitney $U$ test). There are small but statistically significant differences between neurite bundle widths along the $250 \mu \mathrm{m}$ and $500 \mu \mathrm{m}$ rings for both explant types $(* * * \mathrm{p}=$ $1.44 \times 10^{-6}, \mathrm{EphB1}^{-/-} \mathrm{VT} ;{ }^{* *} \mathrm{p}=0.0043, \mathrm{EphB} 1^{-/-} \mathrm{DT}$; Mann Whitney $U$ test). $\mathrm{n}=19$ (DT) and 16 (VT) explants from 3 experiments. (o) CFD of a subset of EphB1 ${ }^{-/-} \mathrm{VT}$ and EphB1 ${ }^{-/-}$DT explants grown on laminin, matched for total neurite outgrowth. EphB1 ${ }^{-/-} \mathrm{VT}$ $\left(\mathrm{n}=7\right.$ explants from 3 experiments) and $\mathrm{EphB1} 1^{-/-} \mathrm{DT}(\mathrm{n}=11$ explants from 3 experiments) explants that had total neurite outgrowth (measured along the $250 \mu \mathrm{m}$ ring) between 120-200 $\mu \mathrm{m}$ were chosen from the full data set. Within this outgrowth-matched subset of explants, EphB1 ${ }^{-/-}$VT neurites form thicker bundles than EphB1 ${ }^{-/-}$DT neurites $\left(* * * \mathrm{p}=2.60 \times 10^{-4}\right.$, $250 \mu \mathrm{m}$ ring; $* * * \mathrm{p}=6.25 \times 10^{-8}, 500 \mu \mathrm{m}$ ring; Mann Whitney $U$ test). Small but statistically significant differences exist between neurite bundle widths along the $250 \mu \mathrm{m}$ and $500 \mu \mathrm{m}$ rings for both explant types in the outgrowth-matched subset of explants $(* * * \mathrm{p}=1.08 \times$ $10^{-4}, \mathrm{EphB} 1^{-/-} \mathrm{VT} ;{ }^{* *} \mathrm{p}=0.0012, \mathrm{EphB} 1^{-/-} \mathrm{DT}$; Mann Whitney $U$ test). Scale bars: $100 \mu \mathrm{m}$ 
(c, d, g), $500 \mu \mathrm{m}$ (f, h). Box and whisker plots show medians with whiskers extending to the minimum and maximum. CFD plots terminate at $3 \mu \mathrm{m}$ along the $\mathrm{x}$-axis for clarity, although data extend to $4+\mu \mathrm{m} . \mathrm{D}=$ dorsal, $\mathrm{V}=$ ventral, $\mathrm{N}=$ nasal, $\mathrm{T}=$ temporal, $\mathrm{M}=$ medial, $\mathrm{L}=$ lateral, $\mathrm{R}=$ rostral, $\mathrm{C}=$ caudal, $\mathrm{dLGN}=$ dorsal lateral geniculate nucleus, $\mathrm{VT}=$ ventrotemporal retina, $\mathrm{DT}=$ dorsotemporal retina, $\mathrm{NF}=$ neurofilament. 


\section{Table 1}

Antibodies Used in Experiments

\begin{tabular}{llll} 
& Antigen & Immunogen & $\begin{array}{l}\text { Manufacturer, species, mono- or polyclonal, } \\
\text { catalog or lot No., RRID }\end{array}$ \\
\hline Primary antibodies & ZsGreen & $\begin{array}{l}\text { Recombinant full-length } \\
\text { Zoanthus } \text { sp. green fluorescent } \\
\text { protein (ZsGreen) }\end{array}$ & $\begin{array}{l}\text { Clontech, rabbit polyclonal, Cat. \# 632474, Lot } \\
\text { \# 1508049, RRID: AB_2491179 }\end{array}$ \\
\hline & Neurofilament & $\begin{array}{l}\text { Membrane preparations from rat } \\
\text { E14-E15, recognizes 165k } \\
\text { protein }\end{array}$ & $\begin{array}{l}\text { Developmental Studies Hybridoma Bank } \\
\text { (DSHB), deposited by T. Jessell and J. Dodd, } \\
\text { mouse monoclonal IgG, Clone 2H3, RRID: } \\
\text { AB_531793 }\end{array}$ \\
\hline Secondary antibodies & Anti-rabbit IgG Alexa Fluor 488 & $\begin{array}{l}\text { Thermo Fisher, goat, Cat. \# A11008, RRID: } \\
\text { AB_143165 }\end{array}$ \\
\hline & Anti-mouse IgG Alexa Fluor 594 & $\begin{array}{l}\text { Thermo Fisher, goat, Cat. \# A11005, RRID: } \\
\text { AB_2534073 }\end{array}$ \\
\hline
\end{tabular}

